\title{
How to determine local stretching and tension in a flow-stretched DNA molecule
}

\author{
Pedersen, Jonas Nyvold; Marie, Rodolphe ; Kristensen, Anders; Flyvbjerg, Henrik
}

Published in:

Physical Review E

Link to article, DOI:

10.1103/PhysRevE.93.042405

Publication date:

2016

Document Version

Publisher's PDF, also known as Version of record

Link back to DTU Orbit

Citation (APA):

Pedersen, J. N., Marie, R., Kristensen, A., \& Flyvbjerg, H. (2016). How to determine local stretching and tension in a flow-stretched DNA molecule. Physical Review E, 93(4), [042405].

https://doi.org/10.1103/PhysRevE.93.042405

\section{General rights}

Copyright and moral rights for the publications made accessible in the public portal are retained by the authors and/or other copyright owners and it is a condition of accessing publications that users recognise and abide by the legal requirements associated with these rights.

- Users may download and print one copy of any publication from the public portal for the purpose of private study or research.

- You may not further distribute the material or use it for any profit-making activity or commercial gain

- You may freely distribute the URL identifying the publication in the public portal

If you believe that this document breaches copyright please contact us providing details, and we will remove access to the work immediately and investigate your claim 


\title{
How to determine local stretching and tension in a flow-stretched DNA molecule
}

\author{
Jonas N. Pedersen, ${ }^{*}$ Rodolphe Marie, Anders Kristensen, and Henrik Flyvbjerg ${ }^{\dagger}$ \\ Department of Micro- and Nanotechnology, Technical University of Denmark, DTU Nanotech, \\ Building 345B, DK-2800 Kongens Lyngby, Denmark \\ (Received 17 September 2015; published 8 April 2016)
}

\begin{abstract}
We determine the nonuniform stretching of and tension in a mega base pairs-long fragment of deoxyribonucleic acid (DNA) that is flow stretched in a nanofluidic chip. We use no markers, do not know the contour length of the DNA, and do not have the full DNA molecule inside our field of view. Instead, we analyze the transverse thermal motion of the DNA. Tension at the center of the DNA adds up to $16 \mathrm{pN}$, giving almost fully stretched DNA. This method was devised for optical mapping of DNA, specifically, DNA denaturation patterns. It may be useful also for other studies, e.g., DNA-protein interactions, specifically, their tension dependence. Generally, wherever long strands of DNA- e.g., native DNA extracted from human cells or bacteria—must be stretched with ease for inspection, this method applies.
\end{abstract}

DOI: 10.1103/PhysRevE.93.042405

\section{INTRODUCTION}

Despite advances in deoxyribonucleic acid (DNA) sequencing technologies in terms of output and cost [1], stretching and imaging of individual long (>100 kbp) DNA molecules remains of interest for complementary sequencing technologies. Such images can, e.g., be used as scaffolds for genome assembly from short reads produced by second-generation sequencing [2] and for detection of structural variations in an ensemble of molecules [2] or even in single molecules [3]. Applications are numerous for micro- and nanofluidic devices capable of stretching DNA to near its contour length. Examples include mapping of restriction sites [2] and the detection of DNA-protein interactions. The latter includes methylation mapping using antibody binding [4], mapping of enzymatic activity [5], and the activity of the replication protein A [6]. Other techniques map the GC content [7], or the denaturation pattern $[3,8]$. For reviews, see, e.g., Refs. $[9,10]$.

DNA molecules can be stretched with molecular combing when DNA is attached to a surface [11], with passive confinement in nanochannels [12-14] or nanoslits [15] and with elongation flows $[16,17]$. Recently, DNA stretched in a nanoslit connecting two microchannels has also received some interest, both experimentally and theoretically $[18,19]$. No flow is applied, but the ends of the DNA molecule extend into the two microchannels, and its midsection in the slit is stretched by the entropic force stemming from the conformational differences between DNA in the nanoslit and in the microchannels.

For all techniques used to stretch DNA, it is imperative to relate the distance measured on images of the DNA to the actual contour length along the DNA. In previous studies of labeled, individual DNA molecules stretched in nanofluidic devices, the degree of stretching was measured either by comparing the length of the DNA in the device to its known contour length $[8,12]$, from the fluorescence intensity of stained molecules $[18,19]$, or by placing markers at known positions along the DNA contour [17]. For the flow-stretch device introduced in Ref. [3] for visualizing DNA molecules

\footnotetext{
*jonas.pedersen@ nanotech.dtu.dk

†henrik.flyvbjerg@ nanotech.dtu.dk
}

longer than $1 \mathrm{Mb}$ (see Fig. 1), the challenge is to estimate the degree of stretching from only a segment of a molecule without knowing its full contour length and without use of markers. As a further complication, the tension is not constant along the DNA. We solve this problem by analyzing the transverse Brownian motion of the molecule. We find that at the center of the slit, the tension in the DNA reaches $16 \mathrm{pN}$, while it decreases to $\sim 0.6 \mathrm{pN}$ near the ends of the DNA. Values for the parallel and perpendicular drag coefficients for DNA in a 85-nm-high nanoslit are also determined (Table I).

Although we here consider the specific problem of extracting the tension in a single DNA molecule from its response to the pressure fluctuations in the liquid around it, the applied method is general. For instance, optical tweezers are calibrated from the Brownian motion of a bead in the trap [20], and the dynamic properties of partially extended DNA molecules stretched by optical tweezers were studied in a similar manner [21]. Finally, the combined effects of confinement and tension on DNA molecules were investigated by pulling at DNA partially confined in a nanoslit [22].

This paper is organized as follows: Section II describes the chip design and the recording of images used for the analysis. Section III gives a qualitative analysis of observations, which leads to the formulation of our model for the transverse thermal motion of the DNA in the nanoslit in Sec. IV. The analytical solution to the dynamics of this model is derived in Sec. V, and the corresponding theoretical power spectrum of transverse displacements is derived in Sec. VI. The recorded positions of the DNA are affected by localization errors due to finite photon statistics, by so-called excess noise from the electronmultiplication process in the electron-multiplying chargecoupled device (EMCCD), and by the motion blur caused by the finite exposure time used in each recorded frame. These effects are discussed in Sec. VII. Experimental power spectral data for transverse displacements of the DNA in the nanoslit are fitted with the theory for these data in Sec. VIII, thus yielding the tension of the DNA in the slit. Fitted values for the drag coefficients of the DNA are compared with estimated values for a cylinder with a similar radius in Sec. IX. Section X discusses the local degree of stretching of the DNA in response to its local tension. Finally, Sec. XI contains the conclusion and discussion. Details and long derivations are found in the Appendix. 

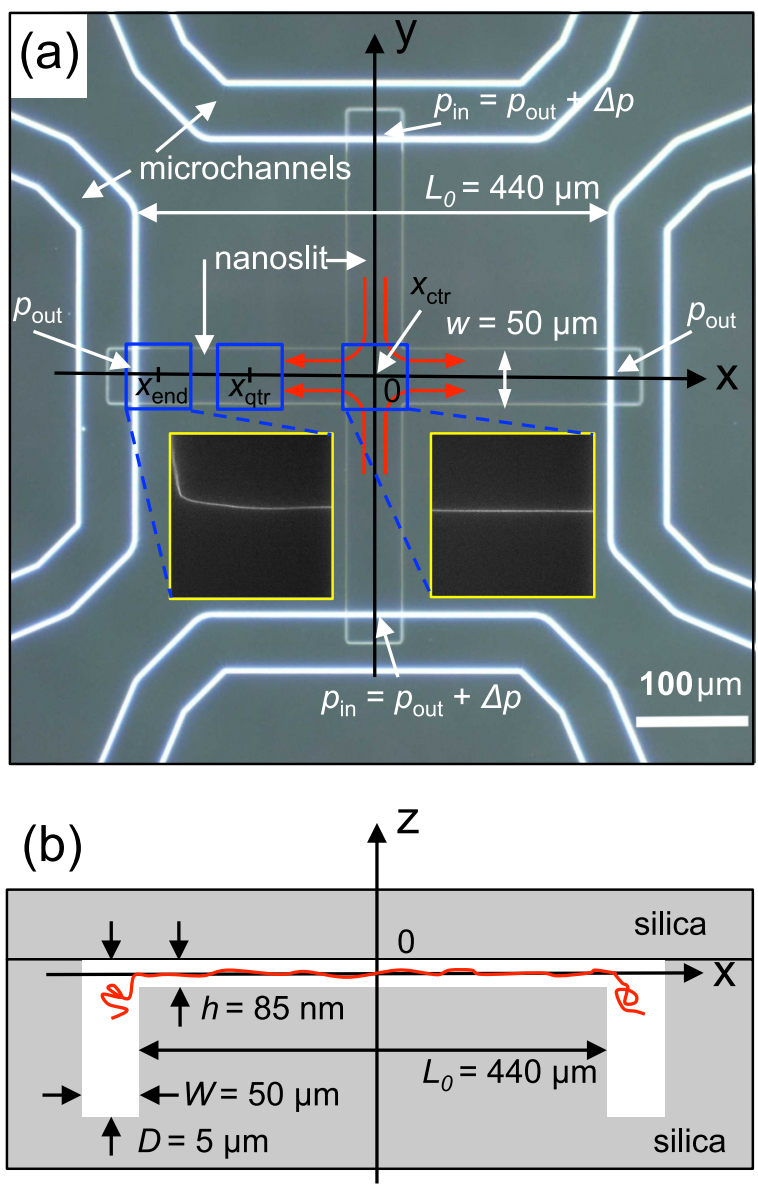

FIG. 1. (a) SEM image of the chip showing a cross-shaped nanoslit connected to four microchannels. A pressure difference $\Delta p$ is applied between the microchannels intersecting the $y$ axis and the microchannels intersecting the $x$ axis. This causes a flow indicated by the red arrows with a stagnation point at the center of the chip. Blue boxes mark the three fields of view used in the analysis of the motion of the DNA. Yellow-edged insets show images of a DNA molecule stretched by the flows. Notice the kink in the DNA at the interface between the nanoslit and the microchannels. (b) Schematics of the cross section of the chip along the $x$ axis (not to scale). The nanoslit is $440 \mu \mathrm{m}$ long and $50 \mu \mathrm{m}$ wide but only $85 \mathrm{~nm}$ high. The microchannels are $5 \mu \mathrm{m}$ deep and $50 \mu \mathrm{m}$ wide. A DNA molecule, stretched between the microchannels, is sketched in red.

\section{CHIP DESIGN AND DATA CAPTURE}

Figure 1(a) shows a SEM image of the chip with its crossshaped nanoslit at the center. The four arms of the nanoslit are

TABLE I. Overview of fitted parameters for each molecule for the drag coefficients perpendicular $\gamma^{\perp}$ and parallel $\gamma^{\|}$to the flow, respectively, and the localization error $\sigma_{\text {pos }}$. Their weighted average (WAVG) are also shown together with values for a cylinder in a flow ("Cyl. Est"), see Secs. IX A and IX B.

\begin{tabular}{lcccc}
\hline \hline & Mol. 1 & Mol. 2 & WAVG & Cyl. Est \\
\hline$\gamma^{\perp}\left[10^{-3} \mathrm{pN} \mathrm{s} /(\mu \mathrm{m})^{2}\right]$ & $1.9 \pm 0.3$ & $2.0 \pm 0.4$ & $1.9 \pm 0.2$ & 3.9 \\
$\gamma^{\|}\left[10^{-3} \mathrm{pN} \mathrm{s} /(\mu \mathrm{m})^{2}\right]$ & $6 \pm 3$ & $7 \pm 3$ & $7 \pm 2$ & 2.1 \\
$\sigma_{\mathrm{pos}}(\mathrm{nm})$ & $43 \pm 1$ & $41 \pm 2$ & $43 \pm 1$ & NA \\
\hline \hline
\end{tabular}

connected to four microchannels. The combined length of two arms of the nanoslit is $L_{0}=440 \mu \mathrm{m}$. Each arm has a width of $w=50 \mu \mathrm{m}$ and a height of only $h=85 \mathrm{~nm}$ [see Fig. 1(b)]. Notice that the depth of the microchannel is $D=5 \mu \mathrm{m} \gg h$.

A pressure $p_{\text {in }}$ is applied to the microchannels connected to the arms of the nanoslit that follow the $y$ axis in Fig. 1 . The microchannels connected to the arms that follow the $x$ axis are kept at a fixed pressure $p_{\text {out }}$. The pressure drop $\Delta p=p_{\text {in }}-p_{\text {out }}$ creates a flow indicated by red arrows in Fig. 1(a). A stagnation point is formed at the center of the nanoslit. For details of the chip fabrication, see Ref. [3]. The same chip design has also been mass produced in polymer material [23,24].

DNA molecules used in the experiment were from stained metaphase chromosomes isolated from human cell culture. The packing of DNA as metaphase chromosomes ensures that long ( $>2$ megabase pair), intact, single DNA molecules are delivered from the pipette tip to the micro- and nanofluidic device without being broken by shear forces [3]. The DNA is uniformly stained with the intercalating dye YOYO-1 (Invitrogen). By proper manipulation of flows, a single, long DNA strand of unknown length is brought from the microchannel into the nanoslit and arranged in the slit such that it spans its whole length from microchannel to microchannel [see Fig. 1(b)].

The DNA is imaged on an inverted microscope (Nikon Eclipse TE2000, Japan) equipped with a $100 \times / 1.4$ oil immersion objective, a $1.5 \times$ optivar lens, and an EMCCD camera (Photometrics Cascade II512, USA) providing a field of view of $55 \times 55 \mu \mathrm{m}^{2}$. The pressure difference $\Delta p$ across the slit is adjusted within 1 mbar to several different values. For each of these values, a movie is recorded for $50 \mathrm{~s}$ at 20 frames per second at three different positions along the device [blue boxes in Fig. 1(a)]: one centered at $x_{\mathrm{ctr}}=0$, another at $x_{\mathrm{qtr}}=-110 \mu \mathrm{m}$, and a third at $x_{\mathrm{end}}=-200 \mu \mathrm{m}$. These movies are used in the subsequent quantitative analysis of the tension in the DNA.

\section{EMPIRICAL FINDINGS AND BASIC CONSIDERATIONS}

The movies recorded at $x_{\mathrm{ctr}}, x_{\mathrm{qtr}}$, and $x_{\mathrm{end}}$ show the DNA as almost fully stretched [yellow-edged insets in Fig. 1(a)]. Especially at the points $x_{\mathrm{ctr}}$ and $x_{\mathrm{qtr}}$, the DNA is almost a straight line, and only transverse fluctuations with a long wavelength, larger than tens of microns, are observed. These fluctuations are thermal motion, caused by pressure fluctuations in the fluid surrounding the DNA. Near the center of the chip, the standard deviations of these fluctuations are less than $1 \mu \mathrm{m}$ (Fig. 2). For comparison, the full length of the nanoslit is $L_{0}=440 \mu \mathrm{m}$.

Near the ends of the nanoslit, on the other hand [see the leftmost yellow-edged inset in Fig. 1(a)], the molecule curves in response to two competing, orthogonal forces and boundary conditions: Where the four arms of the nanoslit meet, the symmetric inflow keeps the DNA molecule in the middle of the two slits with outflow. All along the two arms with outflow, the molecule consequently stays in the middle, because a configuration that intersects flow lines, has higher potential energy in the force field of the flow than one that does not. Moreover, the drag force per unit length of the molecule 


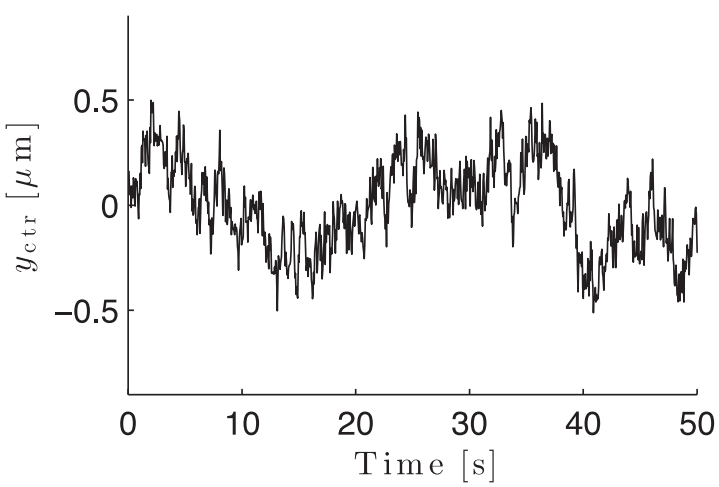

(a)

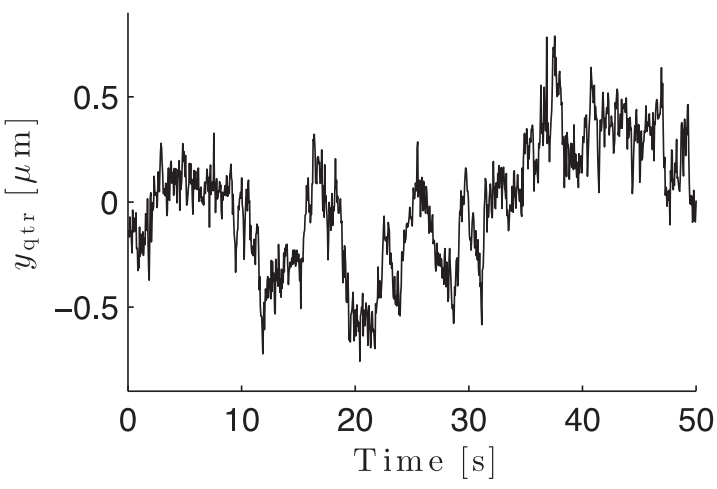

(b)

FIG. 2. The transverse motion of the DNA at the points $x_{\text {ctr }}$ [panel (a)] and $x_{\text {qr }}$ [panel (b)] for an applied pressure $\Delta p=40$ mbar.

is smaller in its longitudinal direction than in its transverse direction, so any part of the molecule that crosses streamlines experiences a torque opposing such crossing. Only near the ends of the arms with outflow-where each arm empties into an orthogonal microchannel-does the DNA in the nanoslit cross stream lines. The force that it takes to do that is the drag force on the DNA segment in the microchannel.

The two parts of the molecule located in the two microchannels move about incessantly in the flows there: A stretched DNA configuration, extending from its point of emergence from the nanoslit and into the microchannel, experiences a torque that will push it towards the microchannel wall, where it experiences a minimum of drag force. It, consequently, coils in response to thermal forces. This brings parts of it away from the channel wall, where stronger flow pulls at the coil to stretch it. The resulting motion is not periodic but random because the thermal coiling is a random process. The resulting motion causes a randomly fluctuating pull on the outermost point of the DNA in the nanoslit. When this pull weakens, the flow in the nanoslit straightens the DNA there. When it becomes stronger, it curves the DNA near the end of the nanoslit. Thus, near the downstream ends of the nanoslit, the DNA's configuration fluctuates in the transverse direction of the slit. The standard deviation of these fluctuations can be up to twice as large as near the center. However, upstream in the nanoslit their amplitude decreases exponentially fast with the distance to the microchannel, and the amplitude vanishes before $x_{\mathrm{qtr}}$, see Appendix D.

A DNA molecule that spans the two nanoslit branches with outflow and reaches into the two microchannels, as sketched in Fig. 1(b), experiences three contributions to its stretching in the nanoslit: (i) in the absence of flow in the microchannels, the free ends of the molecule there can coil thermally to a degree not possible in the nanoslit. This causes a so-called confinement-induced entropic recoil [25], which pulls at both ends of the DNA confined in the nanoslit. This entropic recoil is small compared to the pull from the hydrodynamic drag force in the microchannel, as is obvious from the fact that the flow in the microchannel stretches the DNA there, except towards its ends. But it alone is responsible for stretching the DNA in the absence of flow [26]. (ii) In the presence of flow in the nanoslit, a hydrodynamic drag on the DNA in the nanoslit is present. The tension in the DNA grows in the upstream direction. It grows as the integral over the hydrodynamic drag force on the downstream part of the DNA. So the DNA is stretched the most where the four branches of the nanoslit meet. (iii) Finally, there are fluctuating pulling forces from the hydrodynamic drag forces on the two parts of the molecule in the microchannels. Near the stagnation point in the slit, and most of the way downstream as well, the observed fluctuations of the DNA are caused solely by thermal noise acting on the DNA. The fluctuations in the pull from the microchannels do not contribute to the DNA's motion well inside the nanoslit (see the discussion in Appendix D); there, mainly the average pull from the microchannels is felt.

\section{THE MODEL}

The Kratky-Porod model, also known as the wormlike chain model [27-30], is our starting point for modeling of the DNA in the nanoslit. However, since the DNA is highly stretched, the term containing the persistence length contributes negligibly to the equation of motion for the transverse movement of the DNA (for details, see Appendix A). We consequently omit its persistence length from the model in our calculation of the tension in the DNA and only reintroduce the persistence length when we have determined the tension and need the persistence length in order to calculate the extension of the DNA for the tension we find, see Sec. X. Thus we are left with the usual model of a vibrating string under tension, except our string experiences a space-dependent tension, and its end points at the ends of the nanoslit are not fixed. They move transversely in response to external forces, those on the DNA in the microchannels. Our string also does not vibrate, because its motion is massively overdamped by the viscous fluid that surrounds it. Our string only fluctuates thermally about its average configuration.

With steady flows in the nanoslit and microchannels, the configuration of this string is fully specified at time $t$ by specifying for each point $s$ along its contour, its displacement $(y(s, t), z(s, t))$ orthogonal to the middle line of the slit-arm containing the DNA, see the coordinate system in Fig. 1. Since we model DNA that is almost fully stretched, $s$ does not differ significantly from the $x$ coordinate of the point on the string to which $s$ refers.

The equation of motion for $y(s, t)$ then is

$$
\gamma \partial_{t} y(s, t)=\partial_{s}\left(\tau \partial_{s} y(s, t)\right)+F^{\text {th }}(s, t)
$$

and a similar equation for $z(s, t)$. In these equations, the viscous drag coefficient per unit length $\gamma$ and the tension $\tau$ 


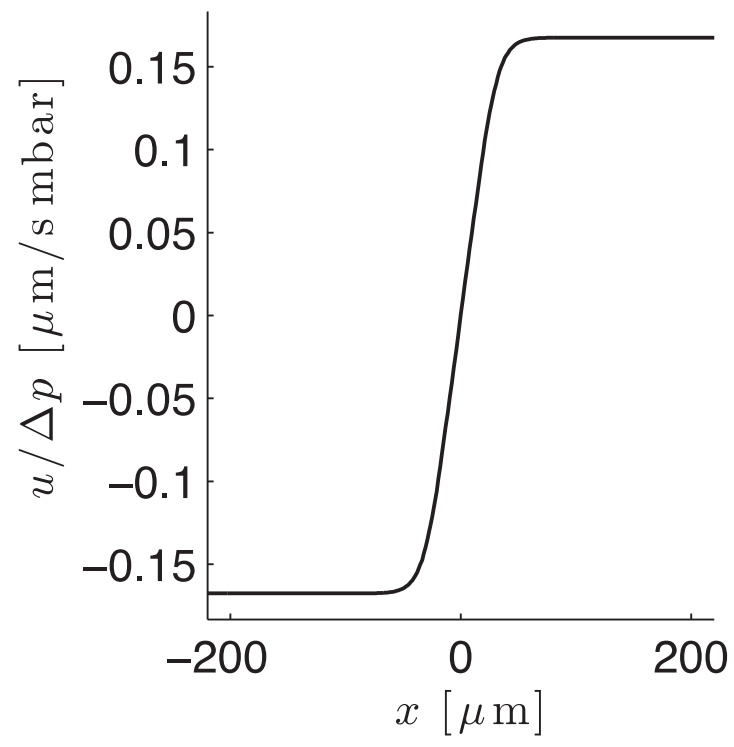

FIG. 3. Flow velocity averaged along the $z$ direction $u$ over the pressure drop along the $x$ axis calculated with COMSOL MULTIPHYSICS for the geometry shown in Fig. 1. The Navier-Stokes equation [Eq. (C1)] is solved under the "Shallow Channel Approximation".

depend on $z$, and $\tau$ on $s$ as well. We cannot observe $z(s, t)$ for the DNA, but we know that it is confined to an 85-nm range, so long-wavelength thermal modes of $z$ are radically suppressed in amplitude. Consequently, their motion self-averages, as do the motions of shorter-wavelength modes, on the time scale of the long-wavelength thermal motion in the $y$ coordinate that we observe. We consequently consider only the effective theory for $y(s, t)$ that results from averaging over $z(s, t)$ locally in space and time, i.e., $s$ and $t$. This effective theory for $y(s, t)$ is reliable, then, only at not-too-short scales of time and space, which is fine with the present experiment, since it does not resolve those scales of space and time due to the diffraction limit and the time averaging (= low-pass filtering) done in video recordings.

In consequence of this $z$-averaged description, $\gamma$ is a constant and $\tau$ depends only on $s$ in Eq. (1). So we introduce $\gamma^{\perp}$ as the viscous drag coefficient per unit length for motion of the string in the $y$ direction, and $F^{\text {th }}(s, t)$ as the stochastic thermal force per unit length of string. It is a generalized Gaussian white-noise density, uncorrelated with itself in space and time: $\left\langle F^{\text {th }}(s, t)\right\rangle=0$ and $\left\langle F^{\text {th }}(s, t) F^{\text {th }}\left(s^{\prime}, t^{\prime}\right)\right\rangle=2 k_{B} T \gamma^{\perp} \delta(s-$ $\left.s^{\prime}\right) \delta\left(t-t^{\prime}\right)$. The quantity $\tau(s)$ is the tension in the string at its point $s$ due to the hydrodynamic drag force on it in the nanoslit and in the microchannels. The boundary conditions on the string's dynamics are that its two ends fluctuate in a prescribed random manner: $y\left(-L_{0} / 2, t\right)=y_{L}(t)$ and $y\left(L_{0} / 2, t\right)=y_{R}(t)$.

A constant pressure drop $\Delta p$ gives a constant flow in the arms of the nanoslit. In each arm, away from the arms's ends and edges, the speed of this laminar flow is independent of $x$ and $y$ in the absence of the DNA molecule (see Fig. 3). We assume that the presence of the DNA does not change this property of the flow pattern well away from the DNA. Now let $u$ denote the $z$-averaged fluid speed experienced by the DNA. It does not depend on $x$ and $y$ well away from the ends and edges of slit arms. As the DNA is almost fully stretched in the flow, the drag force per unit length, $f_{\text {drag }}^{\|}$, from the flow and on a segment $d s$ at the point $s$, is, to a good approximation, independent of the particular conformation of the DNA and proportional to the length of $d s$. More so because our description is an effective one that has been averaged over short times and distances. Consequently, we introduce the effective drag coefficient $\gamma^{\|}$and write the drag force per unit length as proportional to the flow speed $f_{\text {drag }}^{\|}=\gamma^{\|} u$. The proportionality between force and flow speed follows from the fact that the flow has zero Reynolds number-i.e., it is a Stokes flow-even before we average over $z$ and short time scales.

The tension in the DNA at the point $s$ equals the sum of forces pulling at the DNA downstream from $s$. The contribution from the nanoslit to these forces is

$$
\tau_{\text {drag }}(s)=f_{\text {drag }}^{\|}\left(\frac{L_{0}}{2}-|s|\right)=\tau_{\text {nano }}\left(1-\frac{2|s|}{L_{0}}\right),
$$

where $-L_{0} / 2<s<L_{0} / 2$. Here we have introduced $\tau_{\text {nano }}=$ $f_{\text {drag }}^{\|} L_{0} / 2$, which is the contribution from the nanoslit flow to the tension in the DNA at $x_{\mathrm{ctr}}$, where $s=0$ by definition of $s$.

The drag on the DNA in the microchannel gives rise to a fluctuating tension in the DNA in the nanoslit with a static component, its time average, $\tau_{\text {micro }}$. Only this average contributes to the tension in the DNA well inside the slit, beyond the range where the DNA shows transverse motion. This we conclude from the fact that if it were not so, it would result in transverse motion. It also follows from the equation for propagation of transverse waves along a stretched string; see Appendix D. The magnitude of $\tau_{\text {micro }}$ depends on the flow in the microchannel and the amount of DNA there. The latter cannot be obtained from the experiment, so $\tau_{\text {micro }}$ will be a fitting parameter in our data analysis. Adding up, the tension in the DNA in the nanoslit is

$$
\tau(s)=\tau_{\text {micro }}+\tau_{\text {nano }}\left(1-\frac{2|s|}{L_{0}}\right)
$$

at the point $s$. See the black curve in Fig. 4 for an illustration.

Near the ends of the nanoslit, the DNA clearly shows transverse motion. This is due to fluctuations in the pull from the DNA in the microchannels. These fluctuating pulls amount to two fluctuating external forces in the $y$ direction, one on each end point. The competition between these forces and the constant drag force on the DNA in the nanoslit, which counteracts the DNA's crossing of streamlines, results in the motions $y_{L}(t)$ and $y_{R}(t)$ of the two end points at left and right. By treating these motions as boundary conditions on the motion of the DNA in the nanoslit, we find, as follows, that we need not know them [31].

\section{SOLVING THE MODEL}

Since Eq. (1) is linear except for its force term, we can write its solution as a superposition of solutions with different force terms,

$$
y(s, t)=y_{0}(s, t)+y^{\text {th }}(s, t) .
$$

Here $y^{\text {th }}(s, t)$ solves Eq. (1) with the boundary conditions $y^{\text {th }}\left( \pm L_{0} / 2, t\right)=0$ for all $t$, while $y_{0}(s, t)$ is a solution to Eq. (1) for all $t$ without the thermal force $F^{\text {th }}(s, t)$ but with the boundary condition $y_{0}\left( \pm L_{0} / 2, t\right)=y_{L, R}(t)$. Equation (4) 


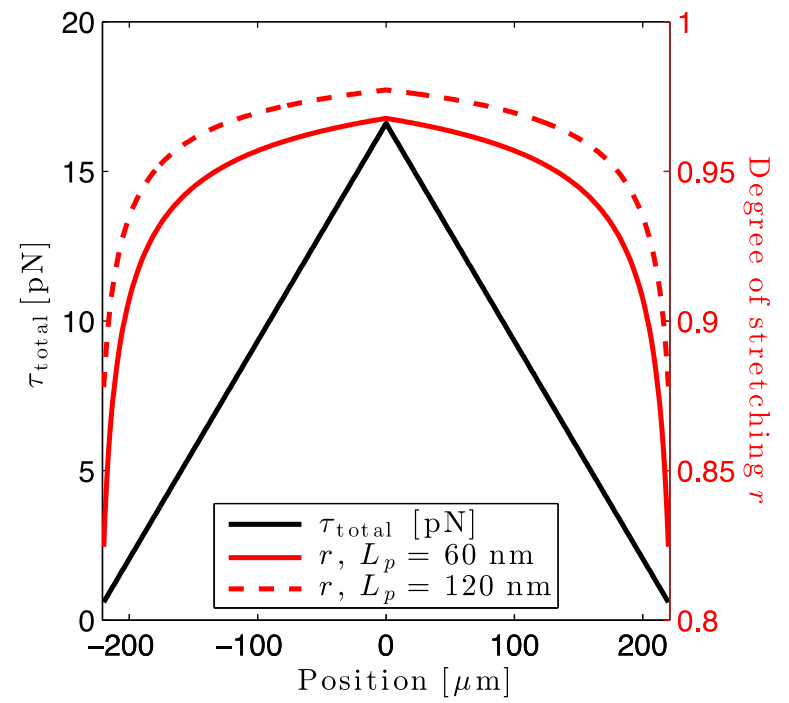

FIG. 4. Black curve, left axis: Total tension $\tau_{\text {total }}$ in the DNA versus the position in the nanoslit from Eq. (3) using the parameters for the tensions $\tau_{\text {micro }}=0.6 \mathrm{pN}$ and $\tau_{\text {nano }}=16 \mathrm{pN}$. The length of the nanoslit is $L_{0}=440 \mu \mathrm{m}$. Red curves, right axis: Corresponding degrees of stretching $r$ calculated with Eq. (26) for the two different values of the persistence length $L_{p}$ discussed in the main text.

solves Eq. (1) with the thermal force $F^{\text {th }}(s, t)$ present and boundary conditions $y\left( \pm L_{0} / 2, t\right)=y_{L, R}(t)$.

\section{A. Fluctuations in pull from microchannels are irrelevant}

The two boundary conditions $y\left( \pm L_{0} / 2, t\right)=y_{L, R}(t)$ can both be split into two: Each can be written as its time-averaged part superposed with its fluctuating part. The time-averaged parts describe the extent to which the DNA on the average is bent away from $y=0$ at the ends of the nanoslit, pulled by the average pull on the DNA in the microchannels from the flows there, in competition with the restoring force caused by the DNA crossing streamlines of the flow in the nanoslit.

The fluctuating parts of the boundary conditions describe how the DNA at the very ends of the nanoslit fluctuate in position in response to fluctuations in the pull on it from the microchannel. These fluctuating positions at the boundary are sources of damped traveling waves in $y(x, t)$ which propagate into the nanoslit from its ends and towards its center. The amplitudes of these two waves decrease exponentially fast with distance from the ends, as do the time-averaged parts. The characteristic "penetration depth" of these waves in the $x$ direction increases with the tension in the DNA.

At all tensions that we find below, these waves are damped to insignificance before the points $x_{\mathrm{qtr}}$ and $x_{\mathrm{ctr}}$, where we record $y(s, t)$ to determine the tension in the string. Details are given in Appendix D. Consequently, $y_{0}(s, t)$ is irrelevant and therefore omitted in the following. With this argument, we have reduced the problem of finding the dynamics of thermally fluctuating DNA with unattached, fluctuating ends and fluctuating tension to the problem of finding the dynamics of thermally fluctuating DNA with attached, unmoving ends and time-invariant tension.

\section{B. Dynamics of thermally fluctuating DNA with fixed ends and time-invariant tension}

Inserting Eq. (3) in Eq. (1), the transverse motion due to thermal noise solves

$$
\begin{aligned}
& \gamma^{\perp} \partial_{t} y^{\text {th }}(s, t)-\left(\tau_{\text {micro }}+\tau_{\text {nano }}\left[1-\frac{|s|}{L_{0} / 2}\right]\right) \partial_{s}^{2} y^{\text {th }}(s, t) \\
& +\frac{\operatorname{sgn}(s) \tau_{\text {nano }}}{L_{0} / 2} \partial_{s} y^{\text {th }}(s, t)=F^{\text {th }}(s, t)
\end{aligned}
$$

with the boundary condition $y^{\text {th }}\left( \pm L_{0} / 2, t\right)=0$ for all $t$. A detailed solution of Eq. (5) is given in Appendix B. The derivation and the main results are sketched here.

Any solution to Eq. (5) can be expanded in an orthogonal basis of eigenfunctions of the spatial operator on the lefthand side of Eq. (5) that satisfy the boundary conditions on $y(s, t)$, i.e., vanish at $s= \pm L_{0} / 2$. With $\gamma^{\perp} \lambda$ the corresponding eigenvalue, the eigenvalue equation reads

$$
\begin{gathered}
-\left(\tau_{\text {micro }}+\tau_{\text {nano }}\left[1-\frac{|s|}{L_{0} / 2}\right]\right) \partial_{s}^{2} A(s) \\
+\frac{\operatorname{sgn}(s) \tau_{\text {nano }}}{L_{0} / 2} \partial_{s} A(s)-\gamma^{\perp} \lambda A(s)=0 .
\end{gathered}
$$

This equation is essentially the time-independent Schrödinger equation for a particle in a wedge-shaped potential. The operator in Eq. (6) is even in $s$, and its eigenfunctions $A_{n}(s)$ are consequently even and odd functions of $s$ and may be numbered by their number of zeros, $n=0,1,2, \ldots$, inside the interval $\left[-L_{0} / 2, L_{0} / 2\right]$. The corresponding spectrum of eigenvalues is discrete, $\lambda_{0}<\lambda_{1}<\lambda_{2}, \ldots$.

For large values of $n, n \gg \sqrt{1+\frac{\tau_{\text {nano }}}{\tau_{\text {micro }}}} / \pi$, the eigenvalues asymptotically become

$$
\sqrt{\lambda_{n}} \simeq \frac{(n+1) \pi / 2}{\sqrt{\gamma^{\perp} L_{0}^{2} \frac{\tau_{\text {micro }}}{\tau_{\text {nano }}^{2}}}\left(\sqrt{1+\frac{\tau_{\text {nano }}}{\tau_{\text {micro }}}}-1\right)}
$$

for $n=0,1,2, \ldots$, with the parity of $n$ giving the parity of its corresponding eigenfunction. For vanishing drag force in the nanoslit, $\tau_{\text {nano }} \rightarrow 0$, one has a string with constant tension $\tau_{\text {micro }}$, and the well-known eigenvalues

$$
\lambda_{n}=\frac{\tau_{\text {micro }}}{\gamma^{\perp}}\left(\frac{[n+1] \pi}{L_{0}}\right)^{2} .
$$

Any solution to Eq. (5) can be written as

$$
y^{\mathrm{th}}(s, t)=\sum_{n=0}^{\infty} y_{n}^{\mathrm{th}}(t) A_{n}(s),
$$

where $y_{n}^{\text {th }}(t)$ satisfies [32]

$$
\partial_{t} y_{n}^{\text {th }}(t)=-\lambda_{n} y_{n}^{\text {th }}(t)+F_{n}^{\text {th }}(t) / \gamma^{\perp} .
$$

Here we introduced

$$
F_{n}^{\mathrm{th}}(t) \equiv \int_{-L_{0} / 2}^{L_{0} / 2} A_{n}(s) F^{\mathrm{th}}(s, t) d s,
$$

which inherit the properties $\left\langle F_{n}(t)\right\rangle=0$ and $\left\langle F_{n}(t) F_{m}\left(t^{\prime}\right)\right\rangle=$ $2 k_{B} T \gamma^{\perp} \delta_{n, m} \delta\left(t-t^{\prime}\right)$ from $F^{\text {th }}(s, t)$. Equation (10) is solved 
by

$$
y_{n}^{\mathrm{th}}(t)=y_{n}^{\mathrm{th}}\left(t_{0}\right) e^{-\lambda_{n}\left(t-t_{0}\right)}+\int_{t_{0}}^{t} e^{-\lambda_{n}\left(t-t^{\prime}\right)} F_{n}^{\mathrm{th}}\left(t^{\prime}\right) / \gamma^{\perp} d t^{\prime},
$$

for given initial time $t_{0}$. Insertion of these solutions in Eq. (9) gives the transverse motion of the DNA at the point $s$ at time $t$. This motion is exactly what we measure in the experiment; see Sec. VI where we define and calculate the power spectrum for the transverse motion of the DNA from Eqs. (9) and (12).

\section{Monte Carlo simulation of the transverse motion}

Images were recorded at discrete times $t_{j}, j=1,2,3, \ldots, N$ with a time lapse $\Delta t=0.05 \mathrm{~s}$ equal to the shutter time and with $N=1000$ frames in the resulting movie. This gives a series of transverse positions $y_{j}^{\text {th }}=y^{\text {th }}\left(s, t_{j}\right)$. Using Eq. (12), $y_{n, j}^{\text {th }} \equiv y_{n}^{\text {th }}\left(t_{j}\right)$ satisfies the dynamic equation

$$
y_{n, j+1}^{\text {th }}=c_{n} y_{n, j}^{\text {th }}+\Delta y_{n, j},
$$

where the notation

$$
\begin{gathered}
c_{n}=e^{-\lambda_{n} \Delta t}, \\
\Delta y_{n, j}=\int_{t_{j}}^{t_{j+1}} e^{-\lambda_{n}\left(t_{j+1}-t\right)} F_{n}^{\mathrm{th}}(t) / \gamma^{\perp} d t,
\end{gathered}
$$

has been introduced, with

$$
\left\langle\Delta y_{n, j}\right\rangle=0 ;\left\langle\Delta y_{n, j} \Delta y_{m, j^{\prime}}\right\rangle=\delta_{n, m} \delta_{j, j^{\prime}} \frac{\left(1-c_{n}^{2}\right)}{\lambda_{n}} \frac{k_{B} T}{\gamma^{\perp}}
$$

by construction. So $\Delta y_{n, j}$ is a Gaussian random variable with expectation value and variance given by Eq. (16).

Finally, a series of values $y^{\text {th }}(s, t)$ is generated by for each mode $n$ creating a series $\left\{y_{n, j}^{\text {th }}\right\}$ from Eq. (13) and inserting the result in Eq. (9).

\section{POWER SPECTRUM ANALYSIS OF THE TRANSVERSE MOTION}

Let the discrete Fourier transformation and its inverse be defined as

$$
\begin{aligned}
& \hat{y}_{k}(s)=\Delta t \sum_{j=1}^{N} y_{j}(s) e^{2 \pi i f_{k} j \Delta t}, k=0,1, \ldots N-1, \\
& y_{j}(s)=\frac{1}{N \Delta t} \sum_{k=0}^{N-1} \hat{y}_{k}(s) e^{-2 \pi i f_{k} j \Delta t}, j=1,2, \ldots N,
\end{aligned}
$$

with $f_{k} \equiv k \Delta f, \Delta f_{k} \equiv 1 / T_{\mathrm{msr}}$, and $T_{\mathrm{msr}} \equiv N \Delta t$. Fourier transformation of Eq. (10) gives an algebraic equation, which is easily solved for $\hat{y}_{k}^{\text {th }}(s)$.

Given a series of measured transverse positions of the DNA $\left\{y_{j}^{\exp }(s)\right\}_{j=1, N}$, the corresponding experimental power spectrum at the point $s$ is defined as

$$
P_{k}^{\exp }(s) \equiv \frac{\left|\hat{y}_{k}^{\exp }(s)\right|^{2}}{T_{\mathrm{msr}}} .
$$

The theoretical values for the averages of the power spectral values are obtained from a Fourier transformation of Eq. (10), leading to an algebraic equation for $\hat{y}_{k}^{\text {th }}(s)$. Using the properties of the noise term and Eq. (9), the averages of the power spectral values become

$$
\begin{aligned}
P_{k}(s) & \equiv \frac{\left\langle\left|\hat{y}_{k}^{\mathrm{th}}(s)\right|^{2}\right\rangle}{T_{\mathrm{msr}}} \\
& =\Delta t \frac{k_{B} T}{\gamma^{\perp}} \sum_{n=0}^{\infty} \frac{1-c_{n}^{2}}{\lambda_{n}} \frac{1}{1+c_{n}^{2}-2 c_{n} \cos (2 \pi k / N)}\left[A_{n}(s)\right]^{2} .
\end{aligned}
$$

That is, the power spectrum is a sum over aliased Lorentzian spectra, with weight factors decreasing with increasing mode number. This is our theory for what we would see, if the experimental time series of positions were series of snapshots taken in an instant compared to the time lapse between shots. This is not the case, however.

\section{MOTION BLUR DUE TO FINITE SHUTTER TIME}

In the operating mode of the EMCCD camera, the shutter is kept open during the time interval $\Delta t$ rather than taking an instant snapshot. This gives a motion-blurred image and the recorded position of the DNA in the nanoslit is the average value during the time interval $\Delta t$. This effect is accounted for by introducing the average position $\bar{y}_{j}(s)$ as the time average of $y(s, t)$ in $\left[t_{j}, t_{j+1}\right]$. Consequently,

$$
\bar{y}_{n, j}=\frac{1}{\Delta t} \int_{t_{j}}^{t_{j+1}} y_{n}^{\mathrm{th}}(t) d t .
$$

By repeating our calculations above in the presence of this time averaging, a power spectrum $\bar{P}_{k}(s)$ for the time-averaged positions is derived,

$$
\begin{aligned}
\bar{P}_{k}(s) \equiv & \frac{\left\langle\left|\hat{\bar{y}}_{k}^{\mathrm{th}}(s)\right|^{2}\right\rangle}{T_{\mathrm{msr}}}=\frac{k_{B} T}{\gamma^{\perp}} \sum_{n=1}^{\infty} \frac{1}{\lambda_{n}^{2}} \\
& \times\left\{\frac{\left(1-c_{n}\right)^{2}}{\lambda_{n} \Delta t c_{n}} \frac{1-c_{n}^{2}}{1+c_{n}^{2}-2 c_{n} \cos \left(\frac{2 \pi k}{N}\right)}\right. \\
& \left.+\left[2-\frac{1}{\lambda_{n} \Delta t} \frac{1-c_{n}^{2}}{c_{n}}\right]\right\}\left[A_{n}(s)\right]^{2} .
\end{aligned}
$$

The effect of the finite shutter time is a suppression of the higher frequencies, i.e., it acts as a low-pass filter. It can be shown that in the limit $\Delta t \rightarrow 0, \bar{P}_{k}(s) \rightarrow P_{k}(s)$. In Monte Carlo simulations, the effect of motion blur can be included by dividing each time step $\Delta t$ into smaller units when simulating the transverse motion and then applying Eq. (21).

Finally, due to the finite number of photons forming the image of the DNA and the stochastic nature of the electronmultiplication process in the EMCCD camera, the transverse position of the DNA obtained from a given image contains a random component,

$$
\bar{y}_{j}^{\mathrm{msr}}(s)=\bar{y}_{j}^{\mathrm{th}}(s)+\sigma_{\mathrm{pos}} \eta_{j},
$$

where $\left\langle\eta_{j}\right\rangle=0$ and $\left\langle\eta_{j} \eta_{j^{\prime}}\right\rangle=\delta_{j, j^{\prime}}$ for all $j$ and $j^{\prime}$. The actual position $\bar{y}_{j}^{\text {th }}(s)$ and the localization error are uncorrelated, so 
the measured power spectrum becomes

$$
\bar{P}_{k}^{\mathrm{msr}}(s) \equiv \frac{\left\langle\left|\hat{\bar{y}}_{k}^{\mathrm{msr}}\right|^{2}\right\rangle}{T_{\mathrm{msr}}}=\bar{P}_{k}(s)+\sigma_{\mathrm{pos}}^{2} \Delta t .
$$

Equation (24) is the expression used to fit experimental data. Since the noise due to the error on position and the noise causing thermal fluctuations are both Gaussian distributed, the measured power spectral values $P_{k}^{\exp }(s)$ are exponentially distributed with expected values $\bar{P}_{k}^{\text {msr }}(s)$, according to theory. This is taken into account by fitting theory to experimental values with maximum likelihood estimation. If the data are consistent with the theory, then the ratios $P_{k}^{\exp }(s) / \bar{P}_{k}^{\mathrm{msr}}(s)$ are exponentially distributed with an expected value equal to 1 . This is used in a Pearson's goodness-of-fit test to calculate $P$ values for the fits.

\section{DATA ANALYSIS}

For each value of the pressure drop $\Delta p$, a time series of the transverse motion is recorded at the positions $x_{\mathrm{ctr}}$ and $x_{\mathrm{qtr}}$ (Fig. 2). The two time series are converted to power spectra (periodigrams) using Eqs. (17) and (19) and fitted with the theoretical power spectra [Eq. (24)] with common values for the fit's parameters at the two different positions $x_{\text {ctr }}$ and $x_{\mathrm{qtr}}$ [not shown]. The parameters are the transverse friction per unit length $\gamma^{\perp}$, the tension due to the drag in the microchannel $\tau_{\text {micro }}$, the tension due to the drag in the nanoslit $\tau_{\text {nano }}$, and the standard deviation $\sigma_{\text {pos }}$ of the localization error on the positions.

Parameter values from the fits of the experimental power spectra were used as input in Monte Carlo simulations to obtain error bars on the fitted parameters without repeating the experiment [see Sec. V C]. That is, we simulated Eq. (13) but included the effects of the finite shutter time and the localization error. The power spectra of the simulated positions were then fitted to Eq. (24), exactly as for the experimental power spectra. The error bars on the parameters fitted to experimental power spectra are the standard deviations of those parameters when fitting to Monte Carlo simulated power spectra.

Error bars are almost as large as the fitted values themselves (except for the localization error). From the consideration leading to the formulation of the model, it is reasonable to assume that the amplitude of the localization error and the value of the friction coefficient $\gamma^{\perp}$ are both independent of the pressure. Consequently, for each molecule we did a new, simultaneous fit to the experimental power spectra for all pressures with common values for $\sigma_{\text {pos }}$ and $\gamma^{\perp}$, see Fig. 5. Error bars on the fitted parameters were obtained as described above.

Within error bars, $\tau_{\text {nano }}$ came out of this fit as proportional to the pressure [Fig. 5(b)]. This it should be, since the velocities of the laminar flows in the nanoslit and microchannel both must be proportional to the pressure according to Stokes equation, and the drag forces on the DNA that cause the tension in it are expected to be proportional to flow velocities. The only possible source of a nonlinear relationship between pressure and tension is the thermal motion of the DNA in the flow, which may give rise to complex motion of the thermally coiled ends of the DNA in the microchannel. However, since a linear

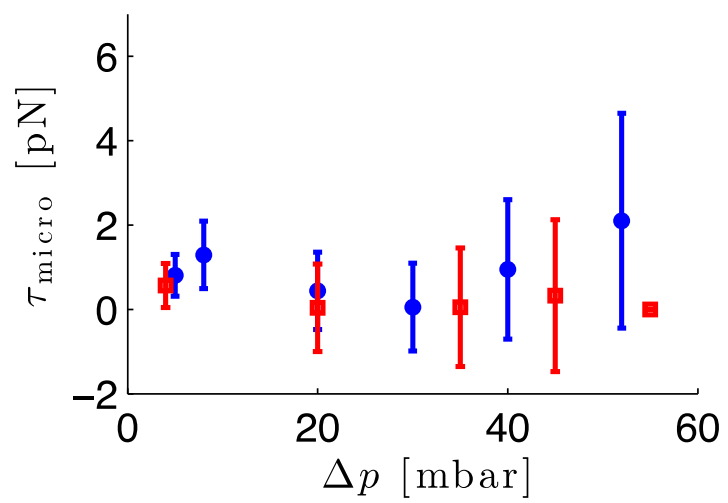

(a)

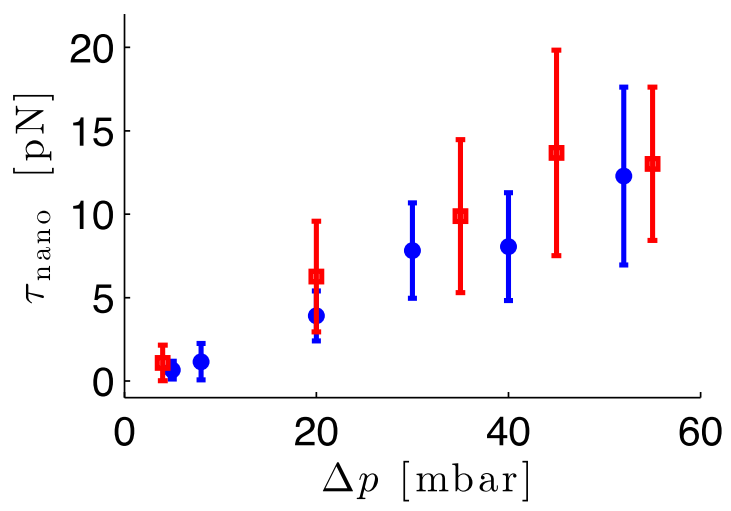

(b)

FIG. 5. Fits of $\tau_{\text {micro }}$ [panel (a)] and $\tau_{\text {nano }}$ [panel (b)] for common values of $\sigma_{\text {pos }}$ and $\gamma^{\perp}$ for two different DNA molecules. $\Delta p$ is the pressure drop, see Fig. 1(a). Error bars are simulated standard deviations of the fitted parameters.

relationship is consistent with our data analysis, we assume this very plausible relationship in yet another simultaneous fit to experimental power spectra at all pressures used, with the constraint that $\tau_{\text {nano }}=a \times \Delta p+b$ imposed. For $b \neq 0$ this relationship is not proportionality but a first-degree polynomial. We kept $b$ in the fit because entropic recoil and a zero-point error in the manometer that measured $\Delta p$, both will cause $b$ to differ from zero, if present. However, simulations confirm that within error bars $b=0$ [not shown], so we did a final fit with the constraint $\tau_{\text {nano }}=a \times \Delta p$. The fit to the power spectra for the pressure drop $\Delta p=40$ mbar is shown as blue curves in Fig. 6 , and the fit parameters $\tau_{\text {nano }}$ and $\tau_{\text {micro }}$ are shown in Figs. 7(a) and 7(b). The total tensions at the center $\tau_{\text {total }}=\tau_{\text {nano }}+\tau_{\text {micro }}$ are shown in Fig. $7(\mathrm{c})$. Error bars are again determined from Monte Carlo simulations. The size of the error bars are comparable to the fitted values themselves, as the fitted variables $a, \tau_{\text {micro }}$ and $\gamma^{\perp}$ are highly correlated. Note also that the data points at different pressures are correlated, as the fit uses common values for the drag coefficient $\gamma^{\perp}$, the localization error $\sigma_{\text {pos }}$, and the relationship $\tau_{\text {nano }}=a \Delta p$.

Table I gives an overview of the fitted parameter values. The weighted average (WAVG) of the fitted transverse friction coefficients per unit length is $\gamma^{\perp}=(1.9 \pm 0.2) \times$ $10^{-3} \mathrm{pNs} /(\mu \mathrm{m})^{2}$. The fits of the tension caused by the flow in the nanoslit including the constraint $\tau_{\text {nano }}=a \Delta p$ are shown in Fig. 7(b). To relate this tension to effective 


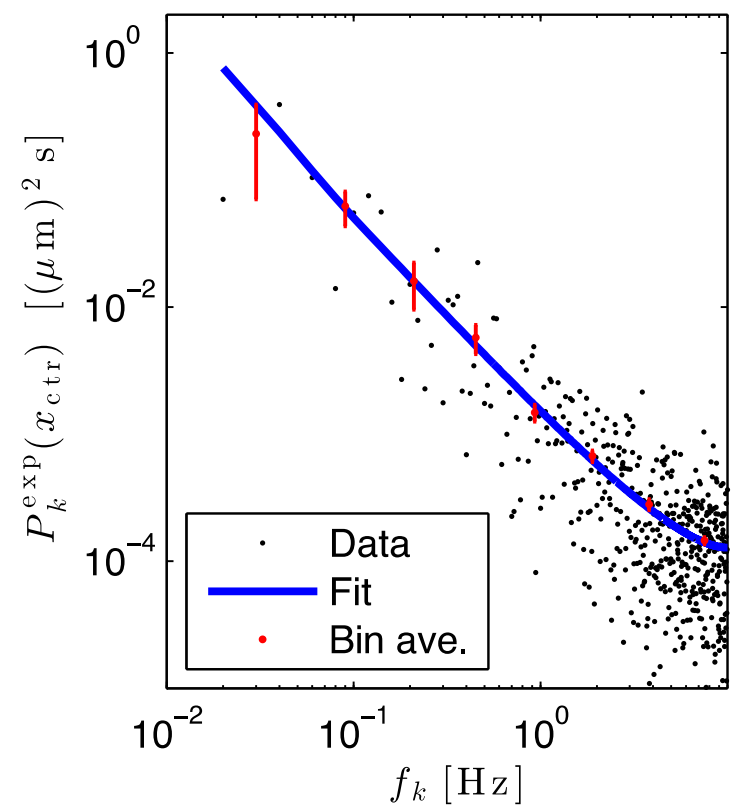

(a)

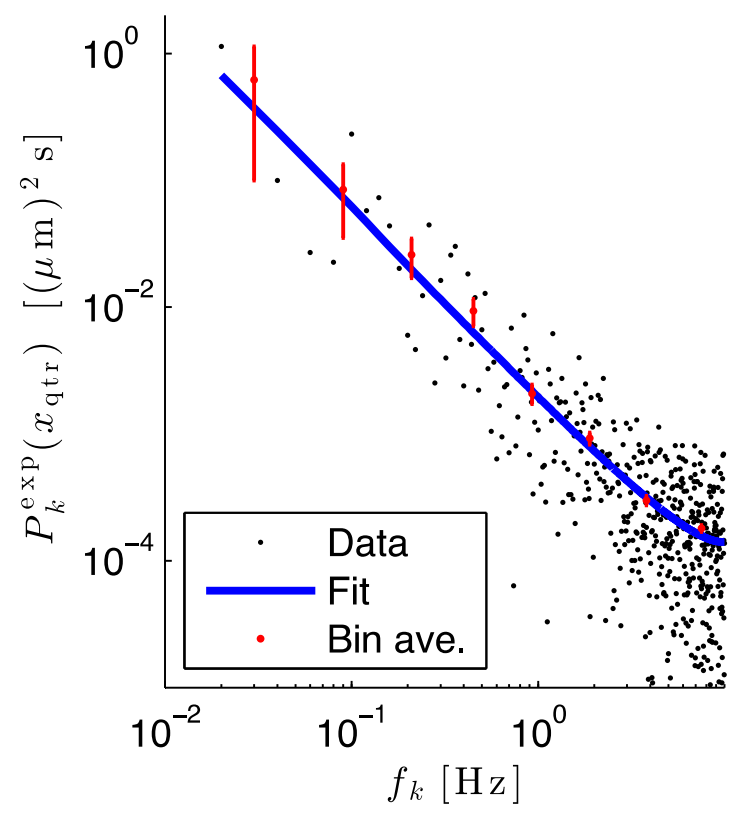

(b)

FIG. 6. Panels (a) and (b) show the power spectra for the time series of the positions recorded at $x_{\mathrm{ctr}}$ and $x_{\mathrm{qtr}}$, respectively, for a pressure drop $\Delta p=40$ mbar. Black dots are the experimental power spectral values $P_{k}^{\exp }(s)$ [Eq. (19)]. Red dots are bin averages with error bars indicating standard errors on the means. Blue lines are fits to Eq. (24) with the constraint that $\tau_{\text {nano }}=a \Delta p$ and common values for the transverse friction coefficient $\gamma^{\perp}$ and the localization error $\sigma_{\text {pos }}$ (see Sec. VIII for details). $P$ values for Pearson's $\chi^{2}$ goodness-of-fit test are 0.77 and 0.23 , respectively.

parallel friction coefficient $\gamma^{\|}$, recall that $\tau_{\text {nano }}=f_{\text {drag }}^{\|} L_{0} / 2=$ $\gamma^{\|} u L_{0} / 2$. A numerical solution of the flow profile in the nanoslit shows that away from the central region the average flow speed is $u=0.17 \mu \mathrm{m} / \mathrm{s}$ mbar (see Appendix C 1 and Fig. 3). Consequently, the weighted average of the effective

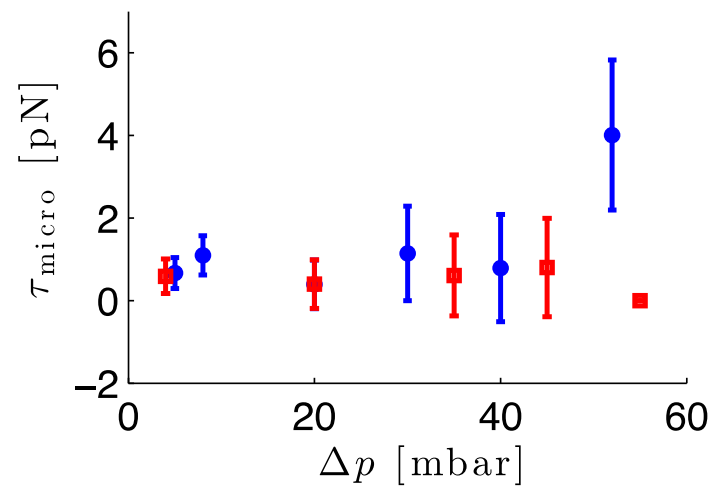

(a)

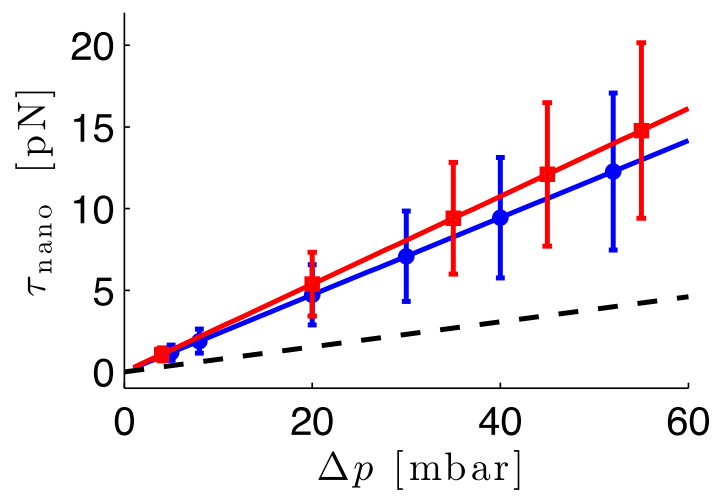

(b)

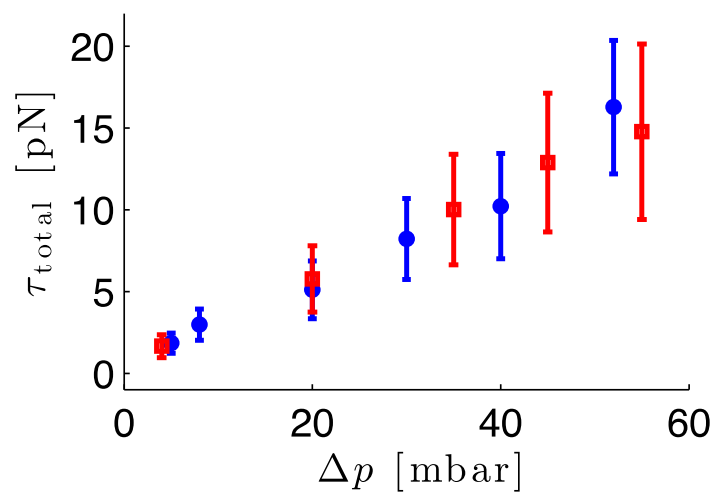

(c)

FIG. 7. Fits for common values of $\sigma_{\text {pos }}$ and $\gamma^{\perp}$ with the constraint that $\tau_{\text {nano }}=a \times \Delta p$ for two different DNA molecules ( $\Delta p$ is the pressure drop). Panel (a) shows $\tau_{\text {micro }}$, panel (b) $\tau_{\text {nano }}$, and panel (c) the total tension at the center of the nanoslit, i.e., $\tau_{\text {total }}=\tau_{\text {micro }}+$ $\tau_{\text {nano }}$. The dashed line in panel (b) marks the tension induced by the estimated drag force on a cylinder with a radius $R_{H}=1 \mathrm{~nm}$ placed at the center of the slit (see Sec. IX). Error bars are simulated standard deviations of the fitted parameters.

friction coefficient per unit length parallel to the flow is $\gamma^{\|}=$ $(7 \pm 2) \times 10^{-3} \mathrm{pNs} /(\mu \mathrm{m})^{2}$. Note that both friction coefficients per unit length are not universal values for DNA but depend on the specific height of the nanoslit. In Sec. IX both values are compared with calculations for a cylinder in a flow.

The standard deviation of the localization error is $\sigma_{\text {pos }}=$ $(43 \pm 1) \mathrm{nm}$, i.e., less than half of the $107-\mathrm{nm}$ pixel width. The fitted values for the tensions due to the flow in the microchannel 
$\tau_{\text {micro }}$ versus the pressure drop, and including the constraint $\tau_{\text {nano }}=a \Delta p$ for the tension in the nanoslit, are shown in Fig. 7(a). Because of the large uncertainties, no clear trends are observed. The weighted average is $\tau_{\text {micro }}=(0.6 \pm 0.3) \mathrm{pN}$ (the value at the highest pressure was omitted).

\section{ESTIMATES FOR THE FITTED PARAMETER VALUES}

In Sec. VIII the drag coefficients per unit length for the motion of the DNA perpendicular and parallel to the flow direction were derived from fits to the power spectra of the transverse motion. In this section, these fitted values are compared with calculations for an infinite cylinder placed in a flow identical to the flow in the arms of the nanoslit device.

\section{A. Perpendicular drag coefficient}

Consider first the friction coefficient per unit length $\gamma^{\perp}$ for DNA moving in the $y$ direction, i.e., orthogonal to the flow with a constant velocity $v_{y}$ (see Fig. 1 ). Now the DNA is approximated by an infinite cylinder with a hydrodynamic radius $R_{H}=1 \mathrm{~nm}$ [33] moving at the center between infinite walls separated by a height $h$ [34]. Faxén derived a formula for the transverse drag force per unit length [35],

$$
\begin{aligned}
\gamma_{\mathrm{drag}}^{\perp}= & \frac{f^{\perp}}{v_{y}} \\
= & 4 \pi \eta /\left[\ln \left(\frac{h}{2 R_{H}}\right)-0.9157\right. \\
& \left.+1.7244\left(\frac{2 R_{H}}{h}\right)^{2}-1.7302\left(\frac{2 R_{H}}{h}\right)^{4}\right],
\end{aligned}
$$

with $\eta$ being the dynamic viscosity of the surrounding liquid. Using $\eta=8.9 \times 10^{-4} \mathrm{~Pa}$ s for water and $h=85 \mathrm{~nm}$ for the height of the nanoslit, the drag coefficient per unit length becomes $\gamma^{\perp}=3.9 \times 10^{-3} \mathrm{pNs} /(\mu \mathrm{m})^{2}$. The analysis based on the transverse motion of the DNA gave a fitted value $\gamma^{\perp}=(1.9 \pm 0.2) \times 10^{-3} \mathrm{pNs} /(\mu \mathrm{m})^{2}$. So the fitted value for DNA and the estimate for a cylinder agree within a factor of 2 .

\section{B. Parallel drag coefficient}

No analytic expression is available for the parallel drag coefficient per unit length $\gamma^{\|}$, so instead we calculate $\gamma^{\|}$ numerically. Details are given in Appendix C 2. As in the previous section, the DNA is approximated by an infinite cylinder with a hydrodynamic radius $R_{H}=1 \mathrm{~nm}$ but now placed along the central axis of an infinite tube with a rectangular cross section and dimensions equal to the arms of the nanoslit (see Fig. 1). The constant pressure drop along the axis is determined in Appendix C 1. The resulting flow profile between the cylinder and the rectangular tube is found numerically. Then the parallel drag force per unit length on the cylinder is computed. The result for the parallel friction coefficient per unit length is $\gamma^{\|}=2.1 \times 10^{-3} \mathrm{pN} \mathrm{s} /(\mu \mathrm{m})^{2}$. This is approximately a factor of 3 smaller than the fitted value for DNA (see Table I).

\section{EXTENSION OF THE DNA}

The maximum extension of the DNA occurs at $x_{\mathrm{ctr}}$, where the total tension is $\tau_{\text {total }}=\tau_{\text {nano }}+\tau_{\text {micro }}$, see Eq. (3). For the range of pressure drops, 5 mbar $<\Delta p<52$ mbar, the total tension spans the range from $2 \mathrm{pN}$ to $16 \mathrm{pN}$; see Fig. 7(c). The local degree of stretching $0<r<1$, where $r$ is the actual length over the contour length, can be related to the local tension $\tau$ by the Marko-Siggia interpolation formula [29], which is very precise for $r$ near 1, where we use it,

$$
\tau=\frac{k_{B} T}{L_{p}}\left[r+\frac{1}{4(1-r)^{2}}-\frac{1}{4}\right] .
$$

Here $L_{p}$ is the persistence length of the DNA. The weighted average of the total tension for the two highest pressures is $16 \pm 3 \mathrm{pN}$. Assuming a persistence length for the stained DNA molecule of $L_{p}=60 \mathrm{~nm}$ in three-dimensional space, the degree of stretching is from $96.4 \%$ to $97.0 \%$ for tensions between $13 \mathrm{pN}$ and $19 \mathrm{pN}$, because $r$ changes slowly with $\tau$ for $r$ near 1 . Since the DNA that we study is close to being restricted to two dimensions, it may be more correct to use the persistence length in two dimensions (2D)that corresponds to the value $60 \mathrm{~nm}$ in 3D, which is $120 \mathrm{~nm}$ [36]. That increases the degree of stretching to the range $97.5 \%$ to $97.9 \%$. We conclude that the issue of whether the DNA should be modeled as constrained to 2D by the slit, is irrelevant, essentially, for our result, as in both cases the DNA is very close to fully stretched. The small uncertainty on the degree of stretching contrasts with the large error bars on the fitted parameter values from which the stretching was derived. This is due to the singular behavior of $\tau$ at $r=1$ and is our good luck. Near the ends of the DNA, the tension is approximately $0.6 \pm 0.3 \mathrm{pN}$, which gives stretchings in the range from $75 \%$ to $86 \%$ for a persistence length of $60 \mathrm{~nm}$, which is consistent with the assumption that the DNA is also almost fully stretched at the entrance of the nanoslit.

The black curve (left axis) in Fig. 4 shows the total tension versus the position in the nanoslit for the fitted value of the tension due to the drag force in the microchannel $\tau_{\text {micro }}=$ $0.6 \mathrm{pN}$, and the maximum value of the tension due to the drag force in the nanoslit $\tau_{\text {nano }}=16 \mathrm{pN}$. The red curves (right axis) show the corresponding degrees of stretching $r$ calculated with Eq. (26) for the two different values of the persistence length discussed above.

\section{CONCLUSION AND DISCUSSION}

Tension in DNA placed in a nanoslit and stretched by an elongation flow was extracted. The analysis showed that the DNA is almost fully stretched ( $~ 97 \%$ of the contour length), much more than by passive confinement in nanochannels or nanoslits. Fitted parameters for the drag coefficients agrees within a factor of 2-3 with simple estimates for a cylinder with similar dimensions.

Alternative methods for extracting the degree of stretching for DNA in nanoslits are, e.g., by use of markers [17] or by comparing the intensity from the stretched part of the stained DNA with the total intensity from it $[18,19]$. The latter requires that the total length of the molecule is known but apparently gives rather large uncertainties in the degree 
of stretching $r$ as the stated values are $r=0.85 \pm 0.16$ and $r=1.06 \pm 0.19$ [19]. Due to the strong nonlinearity of the force-extension relation at large extensions [Eq. (26)], it is difficult to estimate the tension from the degree of stretching.

The method based on the transverse motion of the DNA could in principle be used to determine the tension in DNA molecules spanning a nanoslit and ending in microchannels on both sides with no applied flow $[18,19]$. The analysis is in this case considerably simpler due to the constant tension in the DNA [37]. But for the shorter lengths of the nanoslits used in Refs. $[18,19](<30 \mu \mathrm{m})$, the end-point motion will give nonvanishing contributions to the transverse motion even at the center of the slit. Therefore, much longer slits are required.

For the device in Fig. 1, the results for the degree of stretching of the DNA could in principle be compared with images of barcoded DNA recorded in the same device and aligned with a reference genome [see Ref. [3], Fig. S7(B)]. Despite the qualitative agreement, a full quantitative analysis is challenging as the staining of the DNA with the fluorescent dye YOYO-1 (Invitrogen) changes the contour length of the DNA. This change is known [38], but in practice it is difficult to estimate as the initial staining ratio could be altered by the heat cycle creating the denaturation-renaturation (DR) pattern on the DNA [3].

Finally, in many studies of processes with DNA, e.g., DNA repair [39], it is important to understand the interactions between DNA and proteins. Control of the tension in a single dsDNA molecule has been used to probe such interactions with, e.g., optical tweezers [40] or DNA curtains [41]. These two techniques require that the ends of the dsDNA molecules are functionalized as they are attached to beads or surfaces, respectively. Our device allows both buffer exchange and introduction of reagents, e.g. proteins, to DNA under tension without the need for treatment of the ends and attachment. Thus experiments with megabase-pair-long fragments of native DNA extracted from human cells or bacteria are possible without prior treatment of the DNA. So with the induced nonuniform tension along the DNA (Fig. 4), the flow-stretch design could be used to probe the binding energy of, e.g., DNA intercalators [42] or proteins which induce bending of the DNA $[43,44]$ for various degrees of tension simultaneously.

In addition, nanofluidic devices confine the DNA in a shallow sheet of solution and thus allow for low-background imaging using epifluorescence or total internal reflection fluorescence (TIRF) microscopy as commonly applied in single-molecule experiments [45].

\section{ACKNOWLEDGMENTS}

We thank Kristian E. Jensen for help with the COMSOL MULTIPHYSICS simulations. Funding for this research was provided by the European Union's Seventh Framework Programme FP7/2007-2013 under Grants No. 201418 (READNA) and No. 278204 (Cell-O-Matic); Carlsbergfondet (J.N.P.); Danish Council for Independent Research-Natural Sciences (J.N.P.); and Danish Council for Strategic Research Grant No. 10092322 (PolyNano).

\section{APPENDIX A: WORMLIKE CHAIN MODEL IN THE HIGH-STRETCHING REGIME}

Images of the DNA in the nanoslit show only long wavelength fluctuations, i.e., the DNA is almost fully stretched, so our starting point for the modeling is the wormlike chain (WLC) model in the high-stretching regime. We follow Ref. [29] and use the expression for the free energy to set up the equation of motion for the transverse motion of the DNA and then argue that the term proportional to the persistence length is negligible. These arguments are carried out for a polymer in liquid at rest subjected to a constant force [29], which is not the case for flow-stretched DNA in a nanoslit. As the DNA, however, is almost fully stretched, the tangent vector along the DNA is almost aligned with the flow. Consequently, the torque on each element due to the drag force from the flow is small, so the derivation is still valid in the presence of a flow and almost fully stretched DNA [46].

Let $L_{p}$ denote the persistence length of the polymer and let the polymer be described by a curve in space $\mathbf{r}(s)=$ $\left(r_{x}(s), r_{y}(s), r_{z}(s)\right)$ for $s \in\left[0, L_{c}\right]$, where $L_{c}$ is the contour length of the DNA inside the nanoslit. As the DNA is almost fully stretched, it holds that $L_{c} \simeq L$ where $L$ is the length of the nanoslit. The tangent vector is denoted $\mathbf{t}(s)=\partial_{s} \mathbf{r}(s)$, and as the polymer is unstretchable, $|\mathbf{t}(s)|=1$ for all $s$, i.e., the tangent vector is a unit vector. The curvature is $\kappa(s)=\left|\partial_{s} \mathbf{t}(s)\right|=\left|\partial_{s}^{2} \mathbf{r}(s)\right|$, and the energy cost per length for bending the polymer is $k_{B} T L_{p} \kappa^{2} / 2$.

Consider a constant force $\mathbf{f}=k_{B} T f \hat{\mathbf{x}}$ pulling the polymer in the direction of the $x$ axis. For the WLC model the free energy is

$$
\frac{E}{k_{B} T}=\int_{0}^{L_{c}} d s\left\{\frac{L_{p}}{2}[\kappa(s)]^{2}-\mathbf{f} \cdot \mathbf{t}(s)\right\} .
$$

As the polymer is almost fully stretched, the tangent vector $\mathbf{t}(s)=\left(t_{x}(s), t_{y}(s), t_{z}(s)\right)$ fluctuates only slightly around $\hat{\mathbf{x}}$, i.e., $t_{x}^{2} \gg t_{y}^{2}+t_{z}^{2}=\mathbf{t}_{\perp}^{2}$, where $\mathbf{t}_{\perp}=\left(t_{y}, t_{z}\right)$. Expanding the curvature in powers of the transverse tangent vector and keeping only the quadratic terms, one finds [29], with the use of Eq. (A1), that to quadratic order in $\mathbf{t}_{\perp}$,

$$
\left.\frac{E}{k_{B} T} \simeq \frac{1}{2} \int_{0}^{L_{c}} d s\left\{L_{p}\left[\partial_{s} \mathbf{t}_{\perp}\right]^{2}+f \mathbf{t}_{\perp}^{2}\right]\right\}-f L_{c} .
$$

So to quadratic order in $\mathbf{t}_{\perp}$ the potential energy of the string is

$$
\begin{aligned}
& V_{\mathrm{pot}}\left[\partial_{s} \mathbf{r}_{\perp}(s), \partial_{s}^{2} \mathbf{r}_{\perp}(s)\right] \\
& =\frac{k_{B} T}{2} \int_{0}^{L_{c}} d s\left\{L_{p}\left[\partial_{s}^{2} \mathbf{r}_{\perp}\right]^{2}+f\left[\partial_{s} \mathbf{r}_{\perp}\right]^{2}\right\}-f L_{c},
\end{aligned}
$$

i.e., an integral functional of $\partial_{s} \mathbf{r}_{\perp}$ and $\partial_{s}^{2} \mathbf{r}_{\perp}$. The functional derivative of $V_{\text {pot }}\left[\partial_{s} \mathbf{r}_{\perp}(s), \partial_{s}^{2} \mathbf{r}_{\perp}(s)\right]$ with respect to $r_{y}(s)$ is

$$
\frac{\delta V_{\mathrm{pot}}}{\delta r_{y}(s)}=k_{B} T\left[L_{p} \partial_{s}^{4} r_{y}-f \partial_{s}^{2} r_{y}\right] .
$$

An identical expressions holds for $r_{z}(s)$.

With $\rho$ denoting the mass density of the string, $\gamma$ its viscous drag coefficient per unit length, and $F^{\text {th }}$ the stochastic thermal force per unit length of string, the equation of motion for an 
infinitesimal part of the string at $s$ located at $r_{y}(s)$ is

$$
\rho \ddot{r}_{y}(s, t)=-\frac{\delta V_{\mathrm{pot}}}{\delta r_{y}(s)}-\gamma \dot{r}_{y}(s, t)+F^{\mathrm{th}}(s, t),
$$

which in the overdamped limit becomes

$$
\gamma \dot{r}_{y}(s, t)=k_{B} T\left[-L_{p} \partial_{s}^{4} r_{y}+f \partial_{s}^{2} r_{y}\right]+F^{\text {th }}(s, t) .
$$

We now argue that for our specific problem it holds that $\left|L_{p} \partial_{s}^{4} r_{y}\right| \ll\left|f \partial_{s}^{2} r_{y}\right|$, and, consequently, that the term proportional to $L_{p}$ in Eq. (A6) can be neglected. If the end points are fixed, then $\mathbf{r}_{\perp}(0, t)=\mathbf{r}_{\perp}(L, t)=0$ and the curvature vanishes at the ends, $\partial_{s}^{2} \mathbf{r}_{\perp}(0, t)=\partial_{s}^{2} \mathbf{r}_{\perp}(L, t)=0$. An expansion in eigenmodes $r_{y}(s, t)=\sum_{n=1}^{\infty} r_{y, n}(t) f_{n}(s)$ with $f_{n}(s)=\sqrt{\frac{2}{L}} \sin \left(\frac{n \pi}{L} x\right)(n=1,2, \ldots)$ then gives the equations for the coefficients $r_{y, n}(t)$,

$$
\begin{aligned}
\gamma \partial_{t} r_{y, n}(t)= & -k_{B} T\left[L_{p}\left(\frac{n \pi}{L}\right)^{2}+f\right]\left(\frac{n \pi}{L}\right)^{2} r_{y, n}(t) \\
& +F_{n}^{\mathrm{th}}(t) .
\end{aligned}
$$

We now compare the two terms in the square bracket in Eq. (A7). As the DNA is almost fully stretched, the force $k_{B} T f$ pulling the DNA is of the order of piconewtons. The persistence length is $P \simeq 0.060 \mu \mathrm{m}$, the temperature is $k_{B} T=$ $4.1 \mathrm{fN} \mu \mathrm{m}$, and the length is $L=440 \mu \mathrm{m}$. So the second term is $(n \pi / L)^{2} k_{B} T L_{p} \simeq 10^{-8} n^{2} \mathrm{pN}$. Consequently, neglecting the contribution from the term with the persistence length is reasonable for modes up to $n \sim 10^{4}$, i.e., much higher than the number of modes which can be resolved experimentally.

In conclusion, it is reasonable to drop the term with the persistence length when extracting the tension from the transverse motion of the DNA. For the case of a position-dependent tension this approximation leads to Eq. (1) with $y(s, t)=$ $r_{y}(s, t)$. Note, however, that the persistence length still appears in the expression for the force-extension relation Eq. (26) for the WLC model, because in Eq. (26) the persistence length is an overall factor that sets the scale and dimension.

\section{APPENDIX B: EIGENMODES AND EIGENVALUES}

\section{Solving the eigenvalue equation}

The solution of Eq. (6) is divided into two parts: $A^{<}(s)$ for $s \in\left[-L_{0} / 2,0\right]$, and $A^{>}(s)$ for $s \in\left[0, L_{0} / 2\right]$. Consider first $s>0$. Then the eigenvalue equation becomes

$$
\begin{gathered}
-\left(\tau_{\text {micro }}+\tau_{\text {nano }}\left[1-\frac{s}{L_{0} / 2}\right]\right) \partial_{s}^{2} A^{>}(s) \\
+\frac{\tau_{\text {nano }}}{L_{0} / 2} \partial_{s} A^{>}(s)-\gamma^{\perp} \lambda A^{>}(s)=0 .
\end{gathered}
$$

A series of substitutions brings it on a simpler form. First, we introduce

$$
u=\tau_{\text {micro }}+\tau_{\text {nano }}\left[1-\frac{s}{L_{0} / 2}\right]
$$

which gives

$$
\left[u \partial_{u}^{2}+\partial_{u}+\gamma^{\perp} \lambda\left(\frac{L_{0} / 2}{\tau_{\text {nano }}}\right)^{2}\right] A^{>}(u)=0 .
$$

Then $v=\sqrt{u}$ gives

$$
\left[v^{2} \partial_{v}^{2}+v \partial_{v}+4 \gamma^{\perp} \lambda\left(\frac{L_{0} / 2}{\tau_{\text {nano }}}\right)^{2} v^{2}\right] A^{>}(v)=0,
$$

and, finally, with $K=\sqrt{4 \gamma^{\perp} \lambda\left(\frac{L_{0} / 2}{\tau_{\text {nano }}}\right)^{2}}$ and $q=K v$, the end result is

$$
\left[q^{2} \partial_{q}^{2}+q \partial_{q}+q^{2}\right] A^{>}(q)=0 .
$$

The general solution to Eq. (B5) is the zeroth-order Bessel functions, i.e.,

$$
A^{>}(q)=c_{1} J_{0}(q)+c_{2} Y_{0}(q)
$$

where

$$
q(s)=\sqrt{4 \gamma^{\perp} \lambda\left(\frac{L_{0} / 2}{\tau_{\text {nano }}}\right)^{2} \tau_{\text {micro }}} \sqrt{1+\frac{\tau_{\text {nano }}}{\tau_{\text {micro }}}\left[1-\frac{s}{L_{0} / 2}\right]} .
$$

As $s \in\left[0, L_{0} / 2\right], q(s)$ is always a real number. The boundary condition is $\left.A^{>}(q)\right|_{s=L_{0} / 2}=0$, which is fulfilled for

$$
A^{>}(q)=N\left[Y_{0}\left(\left.q\right|_{s=L_{0} / 2}\right) J_{0}(q)-J_{0}\left(\left.q\right|_{s=L_{0} / 2}\right) Y_{0}(q)\right],
$$

with $N$ being the normalization.

For $s \in\left[-L_{0} / 2,0\right]$ the solution is identical except for the substitution $s \rightarrow-s$.

\section{Matching the solutions}

Due to the symmetry of the force field, it is possible to find a basis of symmetric and antisymmetric solutions [cf. the Schrödinger equation-like form of Eq. (B5) obtained by dividing through with $q^{2}$ ].

\section{a. Symmetric eigenfunctions}

The even solution must fulfill

$$
\partial_{s} A^{<}(s=0)=\partial_{s} A^{>}(s=0)=0,
$$

$$
A^{<}(-s)=A^{>}(s) \text {. }
$$

The solution fulfilling the boundary conditions at $s=$ $\pm L_{0} / 2$ is

$$
\begin{aligned}
A^{>}(q(s))= & N\left\{Y_{0}\left[q\left(L_{0} / 2\right)\right] J_{0}[q(s)]\right. \\
& \left.-J_{0}\left[q\left(L_{0} / 2\right)\right] Y_{0}[q(s)]\right\}, \\
A^{<}(q(s))= & N\left\{Y_{0}\left[q\left(-L_{0} / 2\right)\right] J_{0}[q(-s)]\right. \\
& \left.-J_{0}\left[q\left(-L_{0} / 2\right)\right] Y_{0}[q(-s)]\right\},
\end{aligned}
$$

where $q(s)$ defined in Eq. (B7) depends on the eigenvalue $\lambda$.

The condition on the derivative gives the quantization and determines a series of $\lambda$ s indexed by $n$, which fulfill

$$
\begin{aligned}
0= & N \frac{\sqrt{\lambda_{n} \gamma^{\perp}}}{\sqrt{\tau_{\text {micro }}+\tau_{\text {nano }}}}\left\{Y_{0}\left[q_{n}\left(L_{0} / 2\right)\right] J_{1}\left[q_{n}(0)\right]\right. \\
& \left.-J_{0}\left[q_{n}\left(L_{0} / 2\right)\right] Y_{1}\left[q_{n}(0)\right]\right\},
\end{aligned}
$$

where it is used that $\partial_{s} J_{0}(s)=J_{1}(s)$ and similarly for the other Bessel functions. The solutions to this equation give a 
discrete spectrum of (positive) $\lambda_{n}$ values, which are alternating in magnitude with the solutions to the odd eigenfunctions.

Finally, the normalization is found from

$$
\delta_{n, m}=\int_{-L_{0} / 2}^{L_{0} / 2} d s A_{n}(s) A_{m}(s),
$$

where the orthogonality can be used as a check and $n, m$ denote the different eigenfunctions. For $n=m$ the expression is

$$
\begin{aligned}
1= & 2 N_{n}^{2} \int_{0}^{L_{0} / 2} d s\left[Y_{0}^{2}\left(q_{n}\left(L_{0} / 2\right)\right) J_{0}^{2}\left(q_{n}(s)\right)\right. \\
& +J_{0}^{2}\left(q_{n}\left(L_{0} / 2\right)\right) Y_{0}^{2}\left(q_{n}(s)\right) \\
& -\left\{2 Y_{0}\left(q_{n}\left(L_{0} / 2\right)\right) J_{0}\left(q_{n}\left(L_{0} / 2\right)\right)\right. \\
& \left.\left.\times Y_{0}\left(q_{n}(s)\right) J_{0}\left(q_{n}(s)\right)\right\}\right]
\end{aligned}
$$

This integral is solved numerically.

\section{b. Antisymmetric eigenfunctions}

The odd solution must fulfill

$$
\begin{gathered}
A^{<}(s=0)=A^{>}(s=0)=0, \\
A^{<}(-s)=-A^{>}(s),
\end{gathered}
$$

and the general solutions fulfilling the boundary conditions at $s= \pm L_{0} / 2$ are again given by Eqs. (B11) and (B12). The condition at $s=0$ gives

$$
\begin{aligned}
0= & N\left\{Y_{0}\left[q_{n}\left(L_{0} / 2\right)\right] J_{0}\left[q_{n}(0)\right]\right. \\
& \left.-J_{0}\left[q_{n}\left(L_{0} / 2\right)\right] Y_{0}\left[q_{n}(0)\right]\right\},
\end{aligned}
$$

and the normalization for equal indices is the same as for the even eigenfunctions [see Eq. (B15)], as the only difference is in $\lambda_{n}$.

\section{Asymptotic expressions for the eigenvalues}

The function $q_{n}(s)$ evaluated at $s=0$ and $s=L_{0} / 2$ is

$$
\begin{gathered}
q_{n}(0)=\sqrt{\lambda_{n}} Q \sqrt{1+\frac{\tau_{\text {nano }}}{\tau_{\text {micro }}}}, \\
q_{n}\left(L_{0} / 2\right)=\sqrt{\lambda_{n}} Q,
\end{gathered}
$$

with $Q=\sqrt{\gamma^{\perp}\left(\frac{L_{0}}{\tau_{\text {nano }}}\right)^{2} \tau_{\text {micro }}}$. For complex arguments $z$, the asymptotic expansion for the Bessel functions are for $|z| \rightarrow \infty$ and $|\arg z|<\pi$ [47],

$$
\begin{aligned}
& J_{v}(z)=\sqrt{\frac{2}{\pi z}}\left\{\cos \left(z-\frac{v \pi}{2}-\frac{\pi}{4}\right)+e^{|\operatorname{Im} z|} \mathcal{O}\left(\frac{1}{|z|}\right)\right\}, \\
& Y_{v}(z)=\sqrt{\frac{2}{\pi z}}\left\{\sin \left(z-\frac{v \pi}{2}-\frac{\pi}{4}\right)+e^{|\operatorname{Im} z|} \mathcal{O}\left(\frac{1}{|z|}\right)\right\},
\end{aligned}
$$

i.e., the expansion holds for $\sqrt{\lambda_{n}} Q \gg 1$ [48].

\section{a. Symmetric eigenfunctions}

For the symmetric eigenfunctions, the quantization condition is stated in Eq. (B13). In the asymptotic limit it becomes

$\tan \left(\sqrt{\lambda_{n}} Q-\frac{\pi}{4}\right)=\tan \left(\sqrt{\lambda_{n}} Q \sqrt{1+\frac{\tau_{\text {nano }}}{\tau_{\text {micro }}}}-\frac{3 \pi}{4}\right)$.

This condition is fulfilled when the difference between the arguments equals $n \pi$, where $n=0,1,2, \ldots$ That is,

$$
\sqrt{\lambda_{n}}=\frac{\left(n+\frac{1}{2}\right) \pi}{Q\left(\sqrt{1+\frac{\tau_{\text {nano }}}{\tau_{\text {micro }}}}-1\right)}, \quad n=0,1,2, \ldots
$$

\section{b. Antisymmetric eigenfunctions}

For the antisymmetric eigenfunction the quantization condition is found in Eq. (B18), which in the asymptotic limit becomes

$$
\tan \left(\sqrt{\lambda_{n}} Q-\frac{\pi}{4}\right)=\tan \left(\sqrt{\lambda_{n}} Q \sqrt{1+\frac{\tau_{\text {nano }}}{\tau_{\text {micro }}}}-\frac{\pi}{4}\right) .
$$

The condition again requires that the difference between the arguments is $n \pi$, but now $n=1,2,3, \ldots$ as the zero-eigenvalue corresponds to translation, i.e.,

$$
\sqrt{\lambda_{n}}=\frac{n \pi}{Q\left(\sqrt{1+\frac{\tau_{\text {nano }}}{\tau_{\text {micro }}}}-1\right)}, \quad n=1,2,3, \ldots
$$

\section{c. The full series of asymptotic eigenvalues}

From the analysis above, the result is that for $\sqrt{\lambda_{n}} Q \sim \frac{n \pi}{\sqrt{1+\frac{\tau_{\text {nano }}}{\tau_{\text {micro }}}}-1} \gg 1$ the eigenvalues are

$$
\sqrt{\lambda_{n}}=\frac{(n+1) \pi / 2}{\sqrt{\gamma^{\perp}\left(\frac{L_{0}}{\tau_{\text {nano }}}\right)^{2} \tau_{\text {micro }}}\left(\sqrt{1+\frac{\tau_{\text {nano }}}{\tau_{\text {micro }}}}-1\right)},
$$

for $n=0,1,2, \ldots$. where the parity of $n$ is identical to the parity of the corresponding eigenfunction.

\section{APPENDIX C: FLOW PROFILE}

To compare the fitted values for the parallel and perpendicular drag coefficients per unit length $\left(\gamma^{\|}\right.$and $\left.\gamma^{\perp}\right)$ with estimates for a cylinder in an identical flow, we need to know the flow profile in the nanoslit. Appendix C 1 presents the results of a COMSOL MULTIPHYSICS simulation of the flow profile in the nanoslit shown in Fig. 1. In Appendix C 2 the force on a cylinder placed along the axis of an infinite tube with rectangular cross section is derived.

The starting point is in both cases the Navier-Stokes equation

$$
\frac{\partial \mathbf{v}}{\partial t}+(\mathbf{v} \cdot \nabla) \mathbf{v}=-\frac{1}{\rho} \nabla p+\frac{\eta}{\rho} \nabla^{2} \mathbf{v},
$$

with $\mathbf{v}=\left(v_{x}, v_{y}, v_{z}\right)$ the velocity field. Here $p$ is the pressure, and $\rho=1 \mathrm{~kg} / \mathrm{m}^{3}$ and $\eta=8.9 \times 10^{-4} \mathrm{~Pa}$ s are the density and the dynamic viscosity of water, respectively. A pressure drop of $\Delta p$ is applied between the inlets and the outlets (see Fig. 1), and no-slip boundary conditions are assumed at all walls. 


\section{Flow profile in the nanoslit}

The flow profile for the geometry shown in Fig. 1 was solved numerically with COMSOL MULTIPHYSICS using the "Laminar Flow" module. Instead of solving the full 3D model, the "Shallow Channel Approximation" is used as the height of the channel is only $85 \mathrm{~nm}$. This approximation amounts to adding a volume force $F_{\mathrm{vol}}=-\frac{12 \eta}{h^{2}} \mathbf{v}$ to the right-hand side of the Navier-Stokes equation and solving the resulting 2D model for $v_{x}$ and $v_{y}$. The result is the flow profile averaged along the $z$ direction.

The flow velocity along the $x$ axis is shown in Fig. 3. Away from the stagnation point, the flow reaches a constant speed of $u=0.17(\mu \mathrm{m} / \mathrm{s}$ mbar $) \Delta p$. The pressure gradient is constant away from the central stagnation point and reaches a value of $\frac{\partial p}{\partial x}=2.5 \times 10^{-3}(\mathrm{mbar} / \mu \mathrm{m}) \Delta p$ (not shown). The latter is used as input in Appendix C 2.

These values can be compared with simple estimates: For a channel with length $L_{0}$, a height $h$, infinitely wide, and a pressure drop $\Delta p$, the parabolic flow profile is [49]

$$
v_{x}(z)=\frac{\Delta p}{2 \eta L_{0}}(h / 2-z)(h / 2+z) \text { for }-h / 2<z<h / 2 .
$$

The distance from inlets to the outlets is approximately $L_{0}=440 \mu \mathrm{m}$, and for $h=85 \mathrm{~nm}$ the average speed along the $z$ direction is $u=0.154(\mu \mathrm{m} / \mathrm{s} \mathrm{mbar}) \Delta p$. Close to the result of the full numerical solution. For the pressure gradient the estimate is $\partial p / \partial x \approx \Delta p / L_{0}=2.3 \times 10^{-3}(\mathrm{mbar} / \mu \mathrm{m}) \Delta p$, which is also in good agreement with the full numerical solution.

\section{Drag on a cylinder in the nanoslit}

Consider an infinite cylinder with radius $R_{H}=1 \mathrm{~nm}$ kept at rest at the center and parallel with an infinite tube with a rectangular cross section with height $h=85 \mathrm{~nm}$ and width $w=50 \mu \mathrm{m}$, that is, with dimensions corresponding to the arms of the nanoslit geometry in Fig. 1. A fluid with dynamic viscosity $\eta$ and density $\rho$ flows between the cylinder and the walls of the tube due to a pressure gradient $\nabla p$. The aim is to find the drag force per unit length parallel to the the cylinder due to the fluid flow.

From the Navier-Stokes Eq. (C1), the change in momentum can be cast on the form [50]

$$
\frac{\partial}{\partial t}\left(\rho v_{i}\right)=-\frac{\partial \Pi_{i k}}{\partial x_{k}},
$$

with $\Pi_{i k}$ the momentum flux density tensor (with the dimension force per area). For a viscous fluid, the momentum flux tensor has the form [50]

$$
\Pi_{i k}=p \delta_{i k}+\rho v_{i} v_{k}+\sigma_{i k}^{\prime}=-\sigma_{i k}+\rho v_{i} v_{k}
$$

Here

$$
\sigma_{i k}=-p \delta_{i k}+\sigma_{i k}^{\prime}
$$

is named the stress tensor and $\sigma_{i k}^{\prime}$ the viscous stress tensor. For an incompressible fluid the stress tensor is [50]

$$
\sigma_{i k}=-p \delta_{i k}+\eta\left(\frac{\partial v_{i}}{\partial x_{k}}+\frac{\partial v_{k}}{\partial x_{i}}\right) .
$$

The drag force on a solid surface element (at rest) bounding the fluid is simply the momentum flux through it. For a surface element df the momentum flux through it is [50]

$$
\Pi_{i k} d f_{k}=\left(\rho v_{i} v_{k}-\sigma_{i k}\right) d f_{k} .
$$

The surface element $d f_{k}$ is written on the form $d f_{k}=n_{k} d f$ with $\mathbf{n}$ being a unit vector along the normal. As $\mathbf{v}=0$ at the surface, the force $\mathbf{F}$ acting on a unit surface is

$$
F_{i}=p n_{i}-\sigma_{i k} n_{k},
$$

or, written explicitly in terms of the velocities [Eq. (C6)],

$$
F_{i}=p n_{i}-\eta\left(\frac{\partial v_{i}}{\partial x_{k}}+\frac{\partial v_{k}}{\partial x_{i}}\right) n_{k} .
$$

Due to translational symmetry along the $x$ axis, the flow $\mathbf{v}$ can only have a nonvanishing component in the $x$ direction, and this component is independent of $x$. As we work at low Reynolds number, the term $(\mathbf{v} \cdot \nabla) \mathbf{v}$ is dropped and the timeindependent form of Eq. (C1) becomes

$$
\nabla^{2} v_{x}(x, y)=\frac{1}{\eta} \frac{\partial p}{\partial z}
$$

i.e., a Laplace equation with source term $\frac{1}{\eta} \frac{\partial p}{\partial z}$. Due to translational symmetry along the $x$ axis, $\frac{\partial p}{\partial x}$ must be constant. Equation $(\mathrm{C} 11)$ is solved numerically in COMSOL with $\partial p / \partial x=2.5 \times 10^{-3}(\mathrm{mbar} / \mu \mathrm{m}) \Delta p$ calculated in Appendix $\mathrm{C} 1$ as the source term and the no-slip boundary conditions $v_{x}=0$ at the edges.

The drag force per unit length parallel to the cylinder is

$$
f_{\text {drag }}^{\|}=-\eta \oint d \ell\left(\frac{\partial v_{x}}{\partial y} n_{y}+\frac{\partial v_{x}}{\partial z} n_{z}\right),
$$

as the first term in Eq. (C9) vanishes as $n_{x}=0$, and the last term in the brackets vanishes as $v_{y}=v_{z}=0$ and $v_{x}$ is constant along the $x$ axis. The drag force per unit length on the cylinder due to the fluid is found by integrating Eq. (C11) along the circumference of the cylinder for this solution.

The result is $f_{\text {drag }}^{\|}=3.5 \times 10^{-4}(\mathrm{pN} / \mu \mathrm{m}$ mbar $) \Delta p$. Expressing the drag force per unit length as $f_{\text {drag }}^{\|}=\gamma^{\|} u$, and using $u=0.17(\mu \mathrm{m} / \mathrm{s}$ mbar $) \Delta p$ from Appendix $\mathrm{C} 1$ gives the estimate $\gamma^{\|}=2.1 \times 10^{-3} \mathrm{pN} \mathrm{s} /(\mu \mathrm{m})^{2}$ for the cylinder's parallel drag coefficient per unit length.

\section{APPENDIX D: INFLUENCE OF THE END-POINT MOTION}

In Sec. IV it was stated that for the relevant range of tensions found from the fits to the power spectra, the motion of the end points could be neglected. Here we show how far the end-point motion propagates and that at the points used for the data analysis, i.e., $x_{\mathrm{ctr}}$ and $x_{\mathrm{qtr}}$, the effect on the power spectra is negligible.

\section{Fourier transformation of the end-point motion}

Figure 8 shows an example of the left end-point motion of the DNA and the corresponding power spectrum. For all pressure drops $\Delta p$, the amplitude of the motion is comparable to amplitudes recorded at the positions $x_{\mathrm{ctr}}$ and $x_{\mathrm{qtr}}$. 


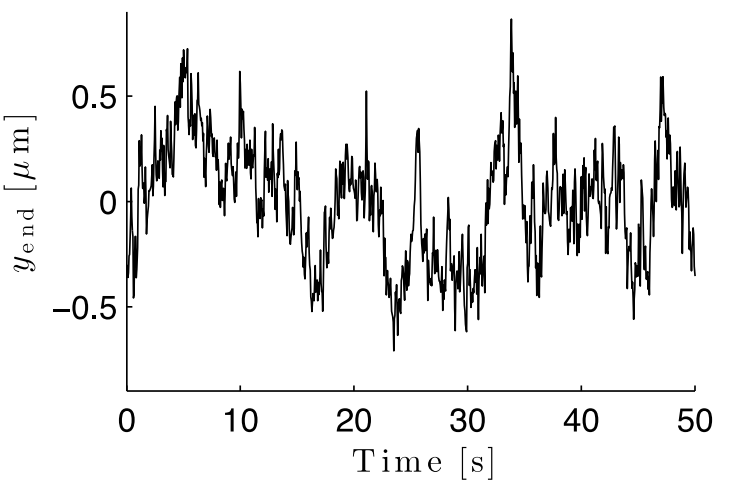

(a)

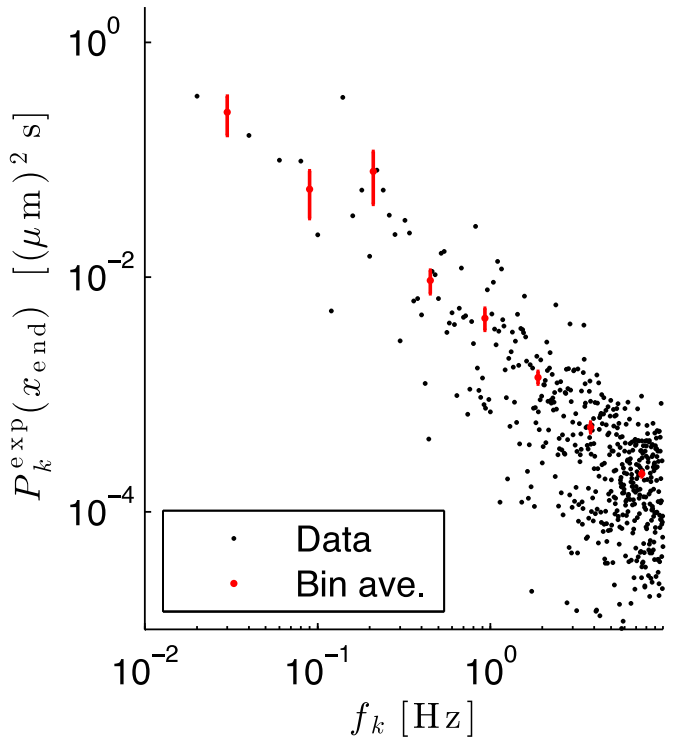

(b)

FIG. 8. Panel (a) shows the transverse motion of the DNA at the position $x_{\text {end }}$ located $20 \mu \mathrm{m}$ from the microchannel for the same DNA molecule as in Fig. 2 and a pressure drop of $\Delta p=40$ mbar. Panel (b) shows the corresponding power spectral values. Error bars are standard errors on the means.

The motion of the left end point is now expressed by its Fourier transform,

$$
y_{L}(t)=\int_{-\infty}^{\infty} d \omega \tilde{y}_{L}(\omega) e^{-i \omega t},
$$

where

$$
\tilde{y}_{L}(\omega)=\frac{1}{2 \pi} \int_{-\infty}^{\infty} d t y_{L}(t) e^{i \omega t} .
$$

The motion in the interior of the string is denoted $y_{0}(s, t)$ with the boundary condition $y_{0}(s, t)=y_{L}(t)$ [see below Eq. (4)]. The motion of the string in the interior, but close to the left end, is assumed to be described by a wave propagating from left to right due to the motion of the string, and we make the ansatz (here $s^{\prime}$ is the distance measured from the left end, $\left.s^{\prime}=s+L_{0} / 2\right)$

$$
y_{0}\left(s^{\prime}, t\right)=\int d \omega \tilde{y}_{L}(\omega) a\left(\omega, s^{\prime}\right) e^{-i \omega t},
$$

with the boundary condition $a\left(\omega, s^{\prime}\right)=1$ to ensure that $y_{0}(0, t)=y_{L}(t)$. As the motion of the end point decays away from the end, the other boundary condition is $a\left(\omega, s^{\prime}\right) \rightarrow 0$ for $s^{\prime} \rightarrow \infty$

\section{Solving for $a(\omega, s)$}

Now we solve the problem of propagation of modes in a string where the tension grows as a first-order polynomial,

$$
\tau\left(s^{\prime}\right)=\tau_{\text {micro }}+\tau_{\text {nano }} \frac{s^{\prime}}{L_{0} / 2} \text { for } \quad s>0 .
$$

That is, we do not seek a solution to the full problem with a tension described by Eq. (3), but a simpler version as we are only interested in the penetration depth of the end-point motion. As we shall see, this motion vanishes before it reaches the center of the slit.

Inserting Eqs. (D3) and (D4) in Eq. (1) and omitting the thermal noise gives the following expression for $a(\omega, s)$,

$$
\begin{aligned}
& -i \omega \gamma^{\perp} a\left(\omega, s^{\prime}\right)-\left(\tau_{\text {micro }}+\tau_{\text {nano }} \frac{s^{\prime}}{L_{0} / 2}\right) \partial_{s}^{2} a\left(\omega, s^{\prime}\right) \\
& -\frac{\tau_{\text {nano }}}{L_{0} / 2} \partial_{s} a\left(\omega, s^{\prime}\right)=0 .
\end{aligned}
$$

Using similar substitutions as in Sec. B 1 leads to

$$
\left[q^{2} \partial_{q}^{2}+q \partial_{q}+q^{2}\right] a(q)=0,
$$

with

$$
\begin{aligned}
q\left(\omega, s^{\prime}\right)= & (1+i) \sqrt{2 \omega \gamma^{\perp}\left(\frac{L_{0} / 2}{\tau_{\text {nano }}}\right)^{2} \tau_{\text {micro }}} \\
& \times \sqrt{1+\frac{\tau_{\text {nano }}}{\tau_{\text {micro }}} \frac{s^{\prime}}{L_{0} / 2}} .
\end{aligned}
$$

The general solution to Eq. (D6) is again a linear combination of zeroth-order Bessel functions [see Eq. (B6)]. From the asymptotic behavior of the Bessel functions for large arguments [see Eqs. (B21) and (B22)], we find that the solution which fulfils the boundary condition $s^{\prime} \rightarrow \infty$ is the zeroth-order Hankel function of the first kind $(z \in \mathbb{C})$

$$
H_{0}^{(1)}(z)=J_{0}(z)+i Y_{0}(z) \text {. }
$$

The final solution, which fulfils the boundary condition $a(q(\omega, 0))=1$, is

$$
a\left(q\left(\omega, s^{\prime}\right)\right)=\frac{H_{0}^{(1)}\left(q\left(\omega, s^{\prime}\right)\right)}{H_{0}^{(1)}(q(\omega, 0))} .
$$

That is, the Fourier coefficient changes from $\tilde{y}_{L}(\omega)$ at the end point to $\tilde{y}_{L}(\omega) a\left(\omega, s^{\prime}\right)$ at $s^{\prime}$, with $a\left(\omega, s^{\prime}\right)$ determined by Eq. (D9), see Eq. (D3).

\section{Power spectrum of the end-point motion}

Assume $y_{0}\left(s^{\prime}, t\right)$ is measured continuously for a time interval $T_{\mathrm{msr}}$. The Fourier transform over the finite interval 
gives the Fourier coefficient

$$
\tilde{y}_{0, k}\left(s^{\prime}\right) \equiv \int_{0}^{T_{\mathrm{msr}}} d \omega e^{2 \pi i f_{k} t} y_{0}\left(s^{\prime}, t\right)=a\left(q\left(\omega_{k}, s^{\prime}\right)\right) y_{L}\left(\omega_{k}\right),
$$

where we used Eq. (D3), $f_{k} \equiv k / T_{\mathrm{msr}}$, and $\omega_{k}=2 \pi f_{k}$.

The experimental power spectrum for the end-point motion at the interior of the string at the point $s^{\prime}$ is defined as

$$
P_{k}^{(\mathrm{ex}, \text { end })}\left(s^{\prime}\right) \equiv \frac{\left|\tilde{y}_{0, k}\left(s^{\prime}\right)\right|^{2}}{T_{\mathrm{msr}}}=\left|a\left(q\left(\omega_{k}, s^{\prime}\right)\right)\right|^{2} \frac{\left|\tilde{y}_{L}\left(\omega_{k}\right)\right|^{2}}{T_{\mathrm{msr}}},
$$

with the expected value

$$
P_{k}^{(\mathrm{end})}\left(s^{\prime}\right)=\left|a\left(q\left(\omega_{k}, s^{\prime}\right)\right)\right|^{2} \frac{\left\langle\left|\tilde{y}_{L}\left(\omega_{k}\right)\right|^{2}\right\rangle}{T_{\mathrm{msr}}} .
$$

Positions are not recorded continuously but at discrete time steps $t_{i}=i \Delta t, i=1, \ldots, N$, with $T_{\mathrm{msr}}=N \Delta t$. So the power spectral values for the frequencies $f_{k}$ will contain aliasing, i.e., contributions from higher frequencies shifted an integer multiple of the sample frequency $f_{\text {sample }}=1 / T_{\mathrm{msr}}$. That is [51]

$$
P_{k}^{(\text {end,aliased })}\left(s^{\prime}\right)=\sum_{n=-\infty}^{\infty} P_{k+n N}^{(\text {end })}\left(s^{\prime}\right) .
$$

Aliasing mainly influences the power spectrum at high frequencies, i.e., near the Nyqvist frequency $f_{\mathrm{Nyq}}=1 / 2 \Delta t$. Here we are only interested in the influence of the end-point motion of the power spectrum recorded at the positions $x_{\mathrm{qtr}}$ and $x_{\mathrm{ctr}}$. As the slowest decaying modes have the lowest frequencies [53], we omit below the influence of aliasing.

Without aliasing the power spectral values decay away from the ends as

$$
\frac{P_{k}^{(\mathrm{end})}\left(s^{\prime}\right)}{P_{k}^{(\mathrm{end})}(0)}=\frac{\left|a\left(q\left(\omega_{k}, s^{\prime}\right)\right)\right|^{2}}{\left|a\left(q\left(\omega_{k}, 0\right)\right)\right|^{2}}=\left|\frac{H_{0}^{(1)}\left(q\left(\omega_{k}, s^{\prime}\right)\right)}{H_{0}^{(1)}\left(q\left(\omega_{k}, 0\right)\right)}\right|^{2} .
$$

The asymptotic expansion of $H_{0}^{(1)}(z)$ for $|z| \rightarrow \infty$ and $-\pi<$ $\arg z<2 \pi$ is [47]

$$
H_{0}^{(1)}(z) \simeq \sqrt{\frac{2}{\pi z}} e^{i(z-\pi / 4)},
$$

so in the asymptotic limit Eq. (D14) becomes

$$
\frac{P_{k}^{(\mathrm{end})}\left(s^{\prime}\right)}{P_{k}^{(\mathrm{end})}(0)} \simeq \frac{2}{\pi\left|q\left(\omega_{k}, s^{\prime}\right)\right|} \frac{e^{-2 \operatorname{Im}\left[q\left(\omega_{k}, s^{\prime}\right)\right]}}{\left|H_{0}^{(1)}\left(q\left(\omega_{k}, 0\right)\right)\right|^{2}} .
$$

For $\frac{\tau_{\text {nano }}}{\tau_{\text {micro }}} \frac{s^{\prime}}{L_{0} / 2} \gg 1$, this expression can be further simplified as $q\left(\omega_{k}, s^{\prime}\right) \simeq(1+i) \sqrt{s^{\prime} / \xi_{k}}$ with $\xi_{k}=\frac{\tau_{\text {nano }}}{\omega_{k} \gamma^{\perp} L_{0}}$, which leads to

$$
\frac{P_{k}^{(\mathrm{end})}\left(s^{\prime}\right)}{P_{k}^{(\mathrm{end})}(0)} \simeq \frac{\sqrt{2}}{\pi\left|H_{0}^{(1)}\left(q_{\omega_{k}}(0)\right)\right|^{2}} \frac{e^{-2 \sqrt{s^{\prime} / \xi_{k}}}}{\sqrt{s^{\prime} / \xi_{k}}} .
$$

Propagation of the end-point motion into the nanoslit increases for increasing tension. As an upper bound we choose $\tau_{\text {nano }}=15 \mathrm{pN}$, see Fig. 7(b). No significant dependence of the pressure drop is observed on the tension due to the drag in the micro channel $\tau_{\text {micro }}$ [Fig. 7(a)]. So we use the weighted average value $0.6 \mathrm{pN}$, see Sec. VIII. The transverse drag coefficient per unit length is set to $\gamma^{\perp}=1.9 \times 10^{-3} \mathrm{pN} \mathrm{s} /(\mu \mathrm{m})^{2}$.

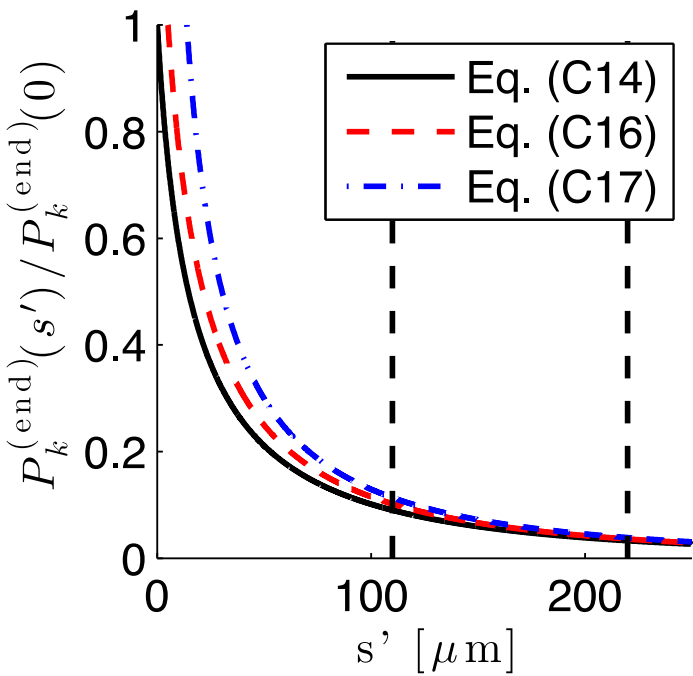

(a)

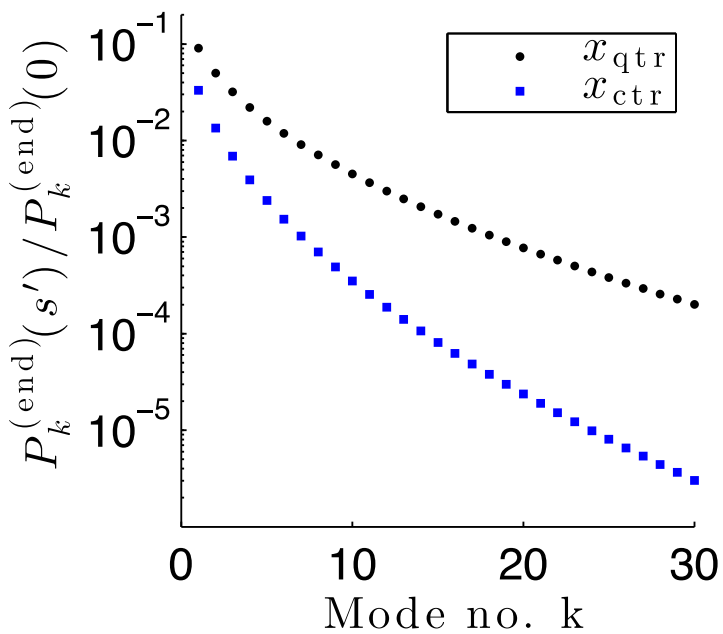

(b)

FIG. 9. Upper bounds for the ratios of the power spectra $P_{k}^{\text {(end) }}\left(s^{\prime}\right) / P_{k}^{(\text {end) }}(0)$. Input parameters are $\tau_{\text {nano }}=15 \mathrm{pN}, \tau_{\text {micro }}=$ $0.6 \mathrm{pN}, \gamma^{\perp}=1.9 \times 10^{-3} \mathrm{pN} \mathrm{s} /(\mu \mathrm{m})^{2}$, and $L_{0}=440 \mu \mathrm{m}$. Panel (a) shows the ratio versus position $s^{\prime}$ for the longest propagating mode, i.e., the mode with $k=1$. The black curve is the exact expression [Eq. (D14)], while the red and the blue curves are the approximations in Eqs. (D16) and (D17), respectively. Panel (b) shows the exact ratio at the two positions $s^{\prime}=x_{\mathrm{qtr}}$ and $s^{\prime}=x_{\mathrm{ctr}}$ used in the analysis of the tension versus the mode number $k$.

The black curve in Fig. 9(a) shows how the ratio $P_{k}^{(\text {end })}\left(s^{\prime}\right) / P_{k}^{(\text {end) }}(0)$ decays versus the position $s^{\prime}$ according to the exact expression in Eq. (D14) for the longest propagating mode, i.e., $k=1$. The blue and the red curves show the results of the two approximations in Eqs. (D16) and (D17), respectively. Vertical lines mark the positions $x_{\mathrm{qtr}}$ and $x_{\mathrm{ctr}}$ where the positions are recorded. Even for the lowest mode, the power spectral value recorded at the position $x_{\mathrm{qtr}}$ is less than $10 \%$ of the value at the end. Figure 9(b) shows how the ratio decays versus mode number at the two different positions. Notice the rapid decay. From the upper bound for the propagation of the end-point motion it is concluded that the end-point motion does not significantly influence the power spectra used for estimating the tension in the string. 
[1] E. R. Mardis, Annu. Rev. Genomics Hum. Genet. 9, 387 (2008).

[2] E. T. Lam, A. Hastie, C. Lin, D. Ehrlich, S. K. Das, M. D. Austin, P. Deshpande, H. Cao, N. Nagarajan, M. Xiao, and P.-Y. Kwok, Nat. Biotechnol. 30, 771 (2012).

[3] R. Marie, J. N. Pedersen, D. L. V. Bauer, K. H. Rasmussen, M. Yusuf, E. Volpi, H. Flyvbjerg, A. Kristensen, and K. U. Mir, Proc. Natl. Acad. Sci. USA 110, 4893 (2013).

[4] S. F. Lim, A. Karpusenko, A. L. Blumers, D. E. Streng, and R. Riehn, Biomicrofluidics 7, 064105 (2013).

[5] C. Vranken, J. Deen, L. Dirix, T. Stakenborg, W. Dehaen, V. Leen, J. Hofkens, and R. K. Neely, Nucl. Acids Res. 42, e50 (2014).

[6] B. Gibb, T. D. Silverstein, I. J. Finkelstein, and E. C. Greene, Anal. Chem. 84, 7607 (2012).

[7] L. K. Nyberg, F. Persson, J. Berg, J. Bergström, E. Fransson, L. Olsson, M. Persson, A. Stålnacke, J. Wigenius, J. O. Tegenfeldt, and F. Westerlund, Biochem. Biophys. Res. Commun. 417, 404 (2012).

[8] W. Reisner, N. B. Larsen, A. Silahtaroglu, A. Kristensen, N. Tommerup, J. O. Tegenfeldt, and H. Flyvbjerg, Proc. Natl. Acad. Sci. U.S.A. 107, 13294 (2010).

[9] R. K. Neely, J. Deen, and J. Hofkens, Biopolymers 95, 298 (2011).

[10] M. Levy-Sakin and Y. Ebenstein, Curr. Opin. Biotechnol. 24, 690 (2013).

[11] A. Bensimon, A. Simon, A. Chiffaudel, V. Croquette, F. Heslot, and D. Bensimon, Science 265, 2096 (1994).

[12] J. O. Tegenfeldt, C. Prinz, H. Cao, S. Chou, W. W. Reisner, R. Riehn, Y. M. Wang, E. C. Cox, J. C. Sturm, P. Silberzan, and R. H. Austin, Proc. Natl. Acad. Sci. U.S.A. 101, 10979 (2004).

[13] W. Reisner, J. N. Pedersen, and R. H. Austin, Rep. Prog. Phys. 75, 106601 (2012)

[14] Y. Kim, K. S. Kim, K. L. Kounovsky, R. Chang, G. Y. Jung, J. J. dePablo, K. Jo, and D. C. Schwartz, Lab Chip 11, 1721 (2011).

[15] K. Jo, D. M. Dhingra, T. Odijk, J. J. de Pablo, M. D. Graham, R. Runnheim, D. Forrest, and D. C. Schwartz, Proc. Natl. Acad. Sci. U.S.A. 104, 2673 (2007).

[16] T. Perkins, D. Smith, and S. Chu, Science 276, 2016 (1997).

[17] R. Dylla-Spears, J. E. Townsend, L. Jen-Jacobson, L. L. Sohn, and S. J. Muller, Lab Chip 10, 1543 (2010).

[18] J.-W. Yeh, A. Taloni, Y.-L. Chen, and C.-F. Chou, Nano Lett. 12, 1597 (2012).

[19] K. L. Kounovsky-Shafer, J. P. Hernández-Ortiz, K. Jo, T. Odijk, J. J. de Pablo, and D. C. Schwartz, Macromolecules 46, 8356 (2013).

[20] K. Berg-Sørensen and H. Flyvbjerg, Rev. Sci. Instrum. 75, 594 (2004).

[21] S. R. Quake, H. Babcock, and S. Chu, Nature 388, 151 (1997).

[22] J. Lin, F. Persson, J. Fritzsche, J. O. Tegenfeldt, and O. A. Saleh, Biophys. J. 102, 96 (2012).

[23] S. Tanzi, P. F. Østergaard, M. Matteucci, T. L. Christiansen, J. Cech, R. Marie, and R. Taboryski, J. Micromech. Microeng. 22, 115008 (2012).

[24] P. F. Østergaard, J. Lopacinska-Jørgensen, J. N. Pedersen, N. Tommerup, A. Kristensen, H. Flyvbjerg, A. Silahtaroglu, R. Marie, and R. Taboryskia, J. Micromech. Microeng. 25, 105002 (2015).
[25] S. W. P. Turner, M. Cabodi, and H. G. Craighead, Phys. Rev. Lett. 88, 128103 (2002)

[26] For a discussion of entropic effects at the micronanofluidic interface, see Refs. [18,19].

[27] O. Kratky and G. Porod, Rec. Trav. Chim. Pays-Bas. 68, 1106 (1949).

[28] C. Bustamante, J. F. Marko, E. D. Siggia, and S. Smith, Science 265, 1599 (1994)

[29] J. Marko and E. Siggia, Macromolecules 28, 8759 (1995).

[30] M. Doi and S. F. Edwards, The Theory of Polymer Dynamics (Oxford University Press, Oxford, 1999).

[31] For a discussion of the role of the end-point motion, see Appendix D.

[32] Note that the equation for $y_{n}^{\text {th }}(t)$ is the equation of motion for a particle in a quadratic potential subjected to a thermal force.

[33] B. S. Fujimoto, J. M. Miller, N. S. Ribeiro, and J. M. Schurr, Biophys. J. 67, 304 (1994).

[34] For our device, the ratio between the height and the width of nanoslit is $h / w=1.7 \times 10^{-3}$, see Fig. 1 .

[35] H. Happel and J. Brenner, Low Reynolds Number Hydrodynamics (Prentice Hall, Upper Saddle River, NJ, 1965).

[36] For studies of the persistence length of DNA in nanoslit confinement, see, e.g., Refs. [54,55].

[37] In contrast to our device, note that in Ref. [19] the width of nanoslit is only $1 \mu \mathrm{m}$, thus hindering the transverse motion of the DNA.

[38] K. Günther, M. Mertig, and R. Seidel, Nucl. Acids Res. 38, 6526 (2010).

[39] I. Heller, T. P. Hoekstra, G. A. King, E. J. G. Peterman, and G. J. L. Wuite, Chem. Rev. 114, 3087 (2014).

[40] J. van Mameren, M. Modesti, R. Kanaar, C. Wyman, E. J. G. Peterman, and G. J. L. Wuite, Nature 457, 745 (2009).

[41] T. Fazio, M.-L. Visnapuu, S. Wind, and E. C. Greene, Langmuir 24, 10524 (2008).

[42] A. S. Biebricher, I. Heller, R. F. H. Roijmans, T. P. Hoekstra, E. J. G. Peterman, and G. J. L. Wuite, Nat. Commun. 6, 7304 (2015).

[43] S. V. Kuznetsov, S. Sugimura, P. Vivas, D. M. Crothers, and A. Ansari, Proc. Natl. Acad. Sci. U.S.A. 103, 18515 (2006).

[44] S. Sugimura and D. M. Crothers, Proc. Natl. Acad. Sci. U.S.A. 103, 18510 (2006).

[45] Y. M. Wang, J. O. Tegenfeldt, W. Reisner, R. Riehn, X.-J. Guan, L. Guo, I. Golding, E. C. Cox, J. Sturm, and R. H. Austin, Proc. Natl. Acad. Sci. U.S.A. 102, 9796 (2005).

[46] Reference [29] considers DNA stretched by a constant force pulling at both ends as well as DNA tethered at one end and stretched by a flow. In the latter case, the DNA is unstretched at the free end, while the DNA in our nanofluidic device is stretched also near the ends of the nanoslit, by the drag forces on the parts of the DNA located in the microchannels.

[47] M. Abramowitz and I. A. Stegun, Handbook of Mathematical Functions: With Formulas, Graphs, and Mathematical Tables (Dover, New York, 1965).

[48] Here $\operatorname{Im} z$ is the imaginary part of $z$.

[49] H. Bruus, Theoretical Microfluidics (Oxford University Press, Oxford, 2007). 
[50] L. Landau and E. M. Lifshitz, Fluid Mechanics (Elsevier Science \& Technology, Amsterdam, 1987)

[51] For a discussion of aliasing, see, e.g., Ref. [52] or Appendix H in Ref. [20].

[52] W. H. Press, Numerical Recipes in Fortran 77: The Art of Scientific Computing (Cambridge University Press, Cambridge, 1992).
[53] For a string with constant tension $\tau$, the amplitude of the endpoint motion decays exponentially with a characteristic length scale $2 \tau /\left(\gamma^{\perp} \omega\right)$.

[54] Y.-L. Chen, P.-k. Lin, and C.-F. Chou, Macromolecules 43, 10204 (2010).

[55] H. W. de Haan and T. N. Shendruk, ACS Macro Lett. 4, 632 (2015). 